\title{
WRINKLING STRUCTURES AT THE RIM OF AN INITIALLY STRETCHED CIRCULAR THIN PLATE SUBJECTED TO TRANSVERSE PRESSURE*
}

\author{
CIPRIAN D. COMAN ${ }^{\dagger}$ AND ANDREW P. BASSOM ${ }^{\ddagger}$
}

Abstract. Short-wavelength wrinkles that appear on an initially stretched thin elastic plate under transverse loading are examined. As the degree of loading is increased so wrinkles appear and their structure at the onset of buckling takes on one of three distinct forms depending on the size of the imposed stretching. With relatively little stretching, the wrinkles sit off the rim of the plate at a location which is not known a priori, but which is determined via a set of consistency conditions. These take the form of constraints on the solutions of certain coupled nonlinear differential equations that are solved numerically. As the degree of stretching grows, so an asymptotic solution of the consistency conditions is possible which heralds the structure that governs a second regime. Now the wrinkle sits next to the rim where its detailed structure can be described by the solution of suitably scaled Airy equations. In each of these first two regimes the Föppl-von Kármán bifurcation equations remain coupled, but as the initial stretching becomes yet stronger the governing equations separate. Further use of singular-perturbation arguments allows us to identify the wavelength wrinkle which is likely to be preferred in practice.

Key words. thin films, wrinkling, Föppl-von Kármán plate equations, asymptotic methods.

AMS subject classifications. $74 \mathrm{G} 10,74 \mathrm{G} 60$

1. Introduction. It is well known that the governing equations for thin rods, plates and shells can be obtained systematically from the general theory of nonlinear elasticity by appealing to suitable asymptotic approximations that exploit the slenderness of such configurations. Typically, the outcome of these reduction schemes is an entire hierarchy of equations rather than a unique set; furthermore, their merit cannot be always gauged a priori and requires a case-by-case appraisal. The Föppl-von Kármán (FvK) nonlinear plate equations were originally derived by ad-hoc approximations but also represent the result of a particular asymptotic reduction (cf. [1], pp.367-447), and have proved to be a versatile choice for describing many interesting phenomena associated with thin elastic films (e.g., [2]). Arguably, this system represents the simplest nonlinear model able to capture the coupling between bending deformations and the in-plane stretching of the plate mid-plane. This approximation, however, does come at at a price and, despite its apparent simplicity, analytical solutions of the FvK system are scarce. The one notable exception is the "Euler column" [3] solution that describes a zero-Gaussian curvature deformation.

The principal aim of the work reported here is to throw light on a number of mathematical structures that have relevance to the FvK bifurcation system. In some recent papers $[4,5,6]$ we have proposed a general asymptotic approach for describing the edge wrinkling experienced by a uniformly stretched circular elastic plate when acted upon by a transverse pressure or a concentrated central load. Generally speaking, the FvK bifurcation system used in our edge-wrinkling investigations is either equivalent to, or based on two coupled nonlinear equations linearised about an ax-

\footnotetext{
* Submitted to the editors February 2, 2018.

†School of Mathematical Sciences, University of Nottingham, University Park, Nottingham NG7 2RD, UK (cdc3p@yahoo.com)

$\ddagger$ School of Physical Sciences, University of Tasmania, Private Bag 37, Hobart TAS 7001, Australia (andrew.bassom@utas.edu.au)
} 
isymmetric nonlinear solution that accounts for the finite mid-plane rotations in the pre-bifurcation state.

The overall picture that emerged in our previous studies is summarised in Figure 1, where typical neutral stability curves $\mathcal{C}_{\mu}$ are illustrated in terms of a non-dimensional transverse loading parameter $\lambda>0$ and the wrinkle mode number (or "wavenumber") $m \in \mathbb{N}$. The driving system also depends on the initial in-plane stretching experienced by the plate, which is described by a dimensionless parameter $\mu$ defined formally in $\S 2$ below. Strictly speaking, it is the case $\mu \gg 1$ that lends itself most naturally to asymptotic analysis since the energy minimum configuration for such problems - the point $\left(m_{c}, \lambda_{c}\right)$ in Figure 1 and corresponding to the least $\lambda$, can be scaled on suitable powers of $\mu$. When $0 \leq \mu \leq \mathcal{O}(1)$ the coordinates of the global minimum of $\mathcal{C}_{\mu}$ are $\mathcal{O}(1)$ quantities, a formal asymptotic strategy breaks down and critical values can only be determined by a direct numerical simulation of the full governing equations. In a strict mathematical sense little can be deduced for $\mu=\mathcal{O}(1)$, but it has been demonstrated in [4] that even then singular perturbation methods can still be used to provide a useful lower bound for the right-hand branch, $\mathcal{C}_{0}^{(+)}$. This is somewhat fortuitous, but proves to be possible because, even though $\lambda$ is formally $\mathcal{O}(1)$, in practice its computed value turns out to be quite large so it can be effectively used as an asymptotically large quantity. We remark that this is an unexpected bonus and cannot be predicted by any formal means. Moreover, as noted in [7], the wrinkling pattern remains strongly localised even when $\mu=0$ although there is no rational theory that might suggest this could have been foreseen.

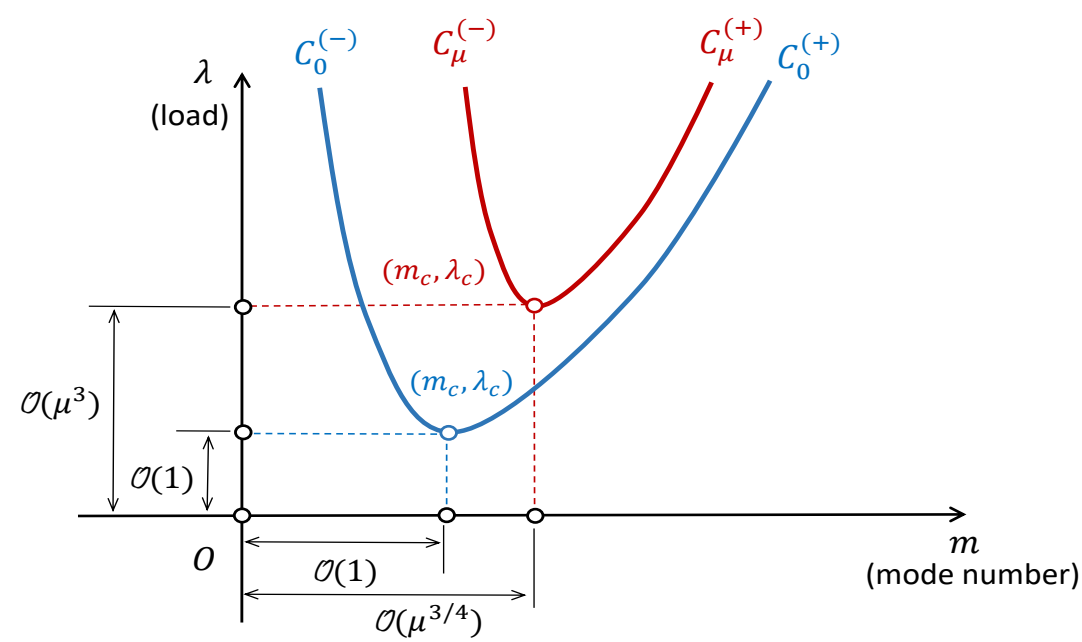

FIG. 1. The features of the neutral stability curves $\mathcal{C}_{\mu}=\mathcal{C}_{\mu}^{(-)} \cup \mathcal{C}_{\mu}^{(+)}$for the initially stretched thin elastic plate subjected to transverse pressure. The vertical axis indicates the non-dimensional pressure $\lambda$ and the abscissa records the mode number $m>0$. The parameter $\mu \geq 0$ represents a non-dimensional measure proportional to the initial degree of radial stretching; thus, the blue curve illustrates an unstretched plate $(\mu=0)$, while the red one corresponds to a taut circular configuration $(\mu \gg 1)$.

Our previous investigations have established that in the limit $\mu \gg 1$ the FvK system decouples and the wrinkling instability is essentially one corresponding to a plane-stress state [8, 9], but with a nonlinear pre-buckling stress distribution. This asymptotic decoupling plays a key role in the success of singular perturbation analyses vis-à-vis the FvK system, a fact that is also implicit in a number of earlier works (e.g., 
see $[10,11])$; for instance, in our problem the decoupled equations are linear and can be solved in closed form to any order (albeit non-trivially). The question remains open as to what happens to the FvK system as $\mu$ is increased from zero (an unstretched plate) to $\mu \gg 1$ (a well-stretched plate), and it is this route to decoupling that motivates our present study.

At this early stage we emphasise that our interest is with the pure buckling problem; that is to determine the nature of $\lambda=\lambda(\mu ; m)$ which is just sufficient to excite wrinkles of wavenumber $m$ for a given $\mu$. The conventional method to isolate the form of $\lambda$ would be to specify $\mu$ and then determine the corresponding $\lambda$ as a function of $m$. What makes this strategy unattractive here is that the basic solution satisfies nonlinear equations that depend on the loading $\lambda$. In standard bifurcation theory one would hope to set the basic state once and for all and then seek eigenvalues of the perturbation equations. Here this approach will fail, or at best be complicated to implement, as the basic equations and the system describing the wrinkles are coupled via $\lambda$. The upshot would be that any critical loading values arising from the wrinkle equations would be likely to modify the base state structure and it is unclear how a converged solution might be arrived at which is consistent with both the base state and wrinkle equations. Fortunately this difficulty can be neatly side-stepped by viewing the problem from a slightly different standpoint. In this we effectively specify $\lambda$, which ties down the base state, and then solve the wrinkle equations for the wavenumber $m$; it is then simple to invert the results to generate the dependence of $\lambda$ on $m$. It is a crucial feature of our work that at no stage is $\lambda$ to be regarded as fixed; rather for a specified $\mu$ we are aiming to track the value of $\lambda(m)$ just sufficient to induce buckling. As the chosen value of $\mu$ is changed so $\lambda$ must compensate to ensure we remain at the onset of buckling.

Within the mechanics of thin plates and shells there are several notable precedents regarding the asymptotic limits of various equations as a loading parameter or a geometrical characteristic is progressively varied. In their pioneering work [12] Junkin and Davis studied a clamped circular plate loaded with a load on a central rigid inclusion by using "first-approximation" non-linear shell equations. Depending on the magnitude of the load, they identified a sequence of plate problems that included the usual linear equations for very small deflections and the FvK equations for moderate deflections. A somewhat similar idea was implemented by Simmonds and Libai [13] for a particular theory of internally pressurised spherical caps. By scaling the pressure load and the shallowness parameters on suitable powers of a dimensionless thickness quantity, they obtained as many as seventeen different types of simplified equations. This suite of equations reflected a range of dominant deformation mechanisms be they linear, nonlinear/inextensional, nonlinear/membrane or some other form. Komaragiri et al. [14] revisited this analysis and carried out a related investigation for a free-standing circular elastic plate under point and pressure loads. In more recent times, Berdichevski's asymptotic-variational technique [15] has emerged as a powerful device that can accomplish comparable results as can be gleaned from [16] among others. It is perhaps worth emphasising that all these studies dealt with deformation problems, that is the load is prescribed and one tries to predict the corresponding deformation. The problem we have in mind is somewhat different as we must tackle a bifurcation equation. Thus, the size of the loading is intimately related to the initial level of stretching, and can only be found by considering both the basic state and the perturbation structure simultaneously.

It is acknowledged that over recent times there has been a plethora of studies concerned with various situations in which wrinkling can arise. Researchers have 
been concerned with developing a comprehensive framework that is able to predict where and how tensional wrinkle patterns evolve. It appears that while many papers deal with stretched plates, relatively few are concerned with the case when wrinkling is provoked by an imposed transverse loading. An excellent survey of some of the key contributions relating to plates subject to stretching or shear has been compiled by Taylor et al. [17] who review the advances that have been made with geometries such as rectangular sheets or circular regions. Our situation is somewhat different in the sense that the wrinkling described below is generated by a transverse pressure load. This is enough to render the basic state genuinely nonlinear and it is then not surprising that the corresponding bifurcation equations are also distinctive. Relatively little effort has been devoted to this class of problems although note should be made of the numerical calculations by Adams [18] who examined the problem of a tensioned circular plate subjected to a concentrated load.

The remainder of the paper is organised in the following way. We begin our study in $\S 2$ with a quick review of the differential equations for the basic state and the linearisation of the FvK system around this solution. A central role in our analysis is played by a suitable large non-dimensional parameter that we shall call $\Delta$, and the paper proceeds by expressing all physical quantities in terms of $\Delta$. In particular, it proves possible to identify the geometry of the right-hand branch of the neutral stability curve and trace its evolution as the original in-plane stretching increases. The nonlinear axisymmetric basic state is revisited in $\S 3$ so that we can reformulate some of the earlier features of [4] in terms of $\Delta$. We also show that for relatively small $\mu$ the associated short-wavelength wrinkle modes are governed by a parabolic cylinder equation which is centred on a point near to, but off the rim of the plate, and whose exact location can only be tied down upon solving a pair of consistency conditions. These are solved numerically in $\S 4$ which shows that the structure of the wrinkles is modified as $\mu$ grows. Indeed, the wrinkles assume an asymptotic form, the key elements of which are outlined in $\S 4.1$. The upshot is that a new modified structure is appropriate to significantly enhanced $\mu$. At this point, which we shall refer to as stage II, the radial extent of the wrinkles has grown but they have also been pushed onto the rim of the plate so that an Airy-type equation becomes the driving form. This stage II structure is developed in $\S 5$, where it is demonstrated how a third regime must take over when $\mu$ is enhanced further. This aspect is taken up in $\S 6$ where it is shown how our asymptotic development automatically captures the identity of the preferred mode when significant in-plane stretching is originally present. The paper closes with some discussion and a few remarks.

2. Formulation. We are interested in the situation depicted in Figure 2 that involves a circular elastic plate of uniform thickness $h>0$ and radius $a$ (with $a / h \gg$ 1 ), a flexurally clamped edge and subjected to a uniform transverse pressure $P$. The deformation of the plate is expressed using a standard cylindrical system of coordinates $(r, \theta, z)$ defined by the usual orthonormal triad $\left\{\boldsymbol{e}_{r}, \boldsymbol{e}_{\theta}, \boldsymbol{e}_{z}\right\}$, with $\boldsymbol{e}_{z}$ perpendicular to the median plane of the plate which also contains the origin of the axes. The linearly elastic material of the plate is characterised by the Youngs' modulus $E>0$ and the Poisson's ratio $0<\nu<1 / 2$.

The starting point for formulating the relevant bifurcation problem is the wellknown Föppl-von Kármán (FvK) system (e.g., see [19]). When written in terms of the transverse displacement $w$ and a suitably defined stress function $F$, these become

$$
D \nabla^{4} w-[F, w]=P \quad \text { and } \quad \nabla^{4} F+\frac{E h}{2}[w, w]=0,
$$



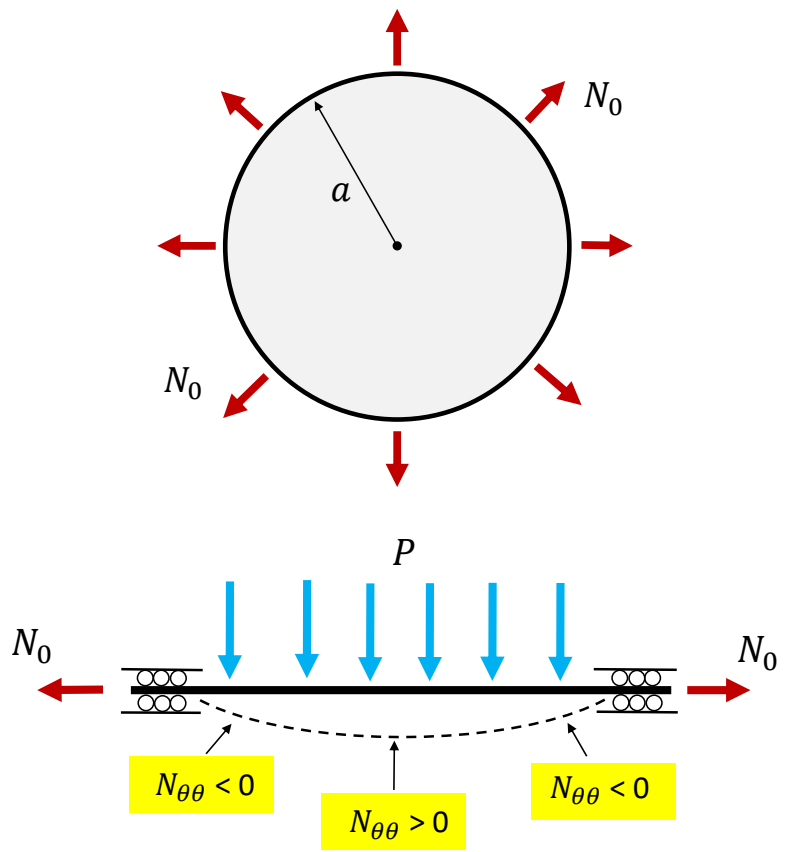

FIG. 2. Top and side views of a uniformly stretched circular thin plate subjected to a uniform transverse pressure; the dashed curve shown above represents its deflected shape.

where the first equation above accounts for the equilibrium in the normal direction, and the second is a compatibility relation expressing the coupling between the Gaussian curvature of the deformed configuration and the membrane stresses. In these equations $D \equiv E h^{3} / 12\left(1-\nu^{2}\right)$ represents the plate bending rigidity, and the bracket denotes the Monge-Ampère bi-linear operator defined by $[f, g]:=\left(\nabla^{2} f\right)\left(\nabla^{2} g\right)-(\nabla \otimes$ $\boldsymbol{\nabla} f):(\boldsymbol{\nabla} \otimes \nabla g)$ for any two smooth functions $f$ and $g$. In addition $F$ is related to the membrane stress tensor $\boldsymbol{N}$ according to $\boldsymbol{N}=\left(\nabla^{2} F\right) \boldsymbol{I}_{2}-\boldsymbol{\nabla} \otimes \boldsymbol{\nabla} F$, where $\boldsymbol{I}_{2}$ is the standard (in-plane) identity tensor $\boldsymbol{I}_{2}=\boldsymbol{e}_{r} \otimes \boldsymbol{e}_{r}+\boldsymbol{e}_{\theta} \otimes \boldsymbol{e}_{\theta}$.

As already mentioned, the plate is clamped in the vertical direction and has normal tractions prescribed along its circumference; this corresponds to

$$
\begin{array}{lll}
w=0, & \frac{\partial w}{\partial r}=0, & \text { on } \quad r=a, \\
N_{r r}=N_{0}, & N_{r \theta}=0, & \text { on } \quad r=a .
\end{array}
$$

To simplify (1) we set $\rho:=r / a$ and introduce the dimensionless quantities

$$
\begin{gathered}
\lambda:=\left[12\left(1-\nu^{2}\right)\right]^{3 / 2}\left(\frac{P}{E}\right)\left(\frac{a}{h}\right)^{4}, \quad \mu^{2}:=12\left(1-\nu^{2}\right)\left(\frac{N_{0}}{E h}\right)\left(\frac{a}{h}\right)^{2}, \\
\bar{w}:=\left[12\left(1-\nu^{2}\right)\right]^{1 / 2} \frac{w}{h}, \quad \bar{F}:=12\left(1-\nu^{2}\right) \frac{F}{E h^{3}}
\end{gathered}
$$

in what follows we shall drop the overbars on these re-scaled variables in order to avoid over-complicating the notation. The parameter $\mu^{2}$ measures the dimensionless bending stiffness In language introduced by Davidovitch et. al [20], $\mu^{2}$ is known as 
the bendability; it is envisaged to be fixed in an experiment while $\lambda$ is increased until wrinkling appears. It can then be shown that for the nonlinear axisymmetric base state the two equations in (1) are reduced to

$$
\mathcal{L}_{0}^{(1)}[\Theta]=\lambda \rho+\frac{\Theta \Phi}{\rho} \quad \text { and } \quad \mathcal{L}_{0}^{(1)}[\Phi]=-\frac{\Theta^{2}}{2 \rho}
$$

where the new dependent variables are $\Theta \equiv \Theta(\rho ; \lambda, \mu):=d w / d \rho$ and $\Phi \equiv \Phi(\rho ; \lambda, \mu):=$ $d F / d \rho$ with $\mathcal{L}_{0}^{(k)}$ denoting the differential operator

$$
\mathcal{L}_{0}^{(k)} \equiv \frac{1}{\rho} \frac{d}{d \rho}\left(\rho \frac{d}{d \rho}\right)-\frac{k^{2}}{\rho^{2}}, \quad(k \in \mathbb{N}) .
$$

The system (4) must be solved subject to the boundary conditions

$$
\Theta(0)=\Theta(1)=\Phi(0)=0, \quad \Phi(1)=\mu^{2} .
$$

2.1. The bifurcation boundary-value problem. As usual, bifurcations from the symmetric basic state (4) are described by a set of equations which follow easily via the method of adjacent equilibrium. This involves considering perturbations to the basic state $w=\stackrel{\circ}{w}(\rho), F=\stackrel{\circ}{F}(\rho)$ which are substituted in the dimensionless version of (1) and then linearized with respect to the incremental fields $\widehat{w} \equiv \widehat{w}(\rho, \theta)$ and $\widehat{F} \equiv \widehat{F}(\rho, \theta)$. The final linear system of partial differential equations is

$$
\nabla^{4} \widehat{w}=[\stackrel{\circ}{w}, \widehat{F}]+[\widehat{w}, \stackrel{\circ}{F}] \quad \text { and } \quad \nabla^{4} \widehat{F}=-[\stackrel{\circ}{w}, \widehat{w}],
$$

which can be simplified further by looking for solutions with separable variables,

$$
(\widehat{w}, \widehat{F})=(W(\rho), \Psi(\rho)) \cos (m \theta),
$$

where $m \geq 0$ is an arbitrary integer at this stage. The unknown amplitudes in (7) satisfy the linear system

$$
\mathcal{L}_{11}[W]+\mathcal{L}_{12}[\Psi]=0 \quad \text { and } \quad \mathcal{L}_{21}[W]+\mathcal{L}_{22}[\Psi]=0,
$$

where we have introduced the ordinary differential operators

$$
\begin{gathered}
\mathcal{L}_{11} \equiv\left[\mathcal{L}_{0}^{(m)}\right]^{2}-\frac{1}{\rho} \frac{d}{d \rho}\left(\Phi \frac{d}{d \rho}\right)+\frac{d \Phi}{d \rho}\left(\frac{m}{\rho}\right)^{2}, \quad \mathcal{L}_{22} \equiv\left[\mathcal{L}_{0}^{(m)}\right]^{2} \\
\mathcal{L}_{12}=-\mathcal{L}_{21} \equiv-\frac{1}{\rho} \frac{d}{d \rho}\left(\Theta \frac{d}{d \rho}\right)+\frac{d \Theta}{d \rho}\left(\frac{m}{\rho}\right)^{2}
\end{gathered}
$$

This eighth-order system is to be solved subject to suitable regularity conditions at the centre of the plate together with the rim conditions (2) appropriate for a flexurally clamped plate. In dimensionless form these constraints become simply

$$
W=\frac{d W}{d \rho}=\Psi=\frac{d \Psi}{d \rho}=0, \quad \text { for } \quad \rho \in\{0,1\} .
$$

Our stated intention with this work is to explore the behaviour of the FvK system over the entire range of values of $\mu \in[0, \infty)$ that measures the initial in-plane stretching of the plate. Guided by our earlier remark, that even when $\mu$ is small 
the eigenvalue $\lambda$ tends to be large, it is convenient to introduce the auxiliary fixed non-dimensional parameter $\Delta \gg 1$. The strategy we shall adopt is to monitor the behaviour of the system by using various quantities expressed in terms of the $\Delta$. In particular results developed in [6] showed that when the wavenumber $m$ is large the corresponding critical loading required for wrinkling occurs at a value $\lambda \propto m^{8 / 3}$. Guided by this we write

$$
\lambda=\lambda_{0} \Delta^{4}, \quad \lambda_{0}=\mathcal{O}(1)
$$

together with the squared mode number

$$
m^{2}=M_{0} \Delta^{3}+M_{1} \Delta^{11 / 4}+M_{2} \Delta^{5 / 2}+\ldots, \quad M_{j}=\mathcal{O}(1), \quad(j=0,1,2) .
$$

We remark that we could subsume the quantity $M_{0}$ within the definition of $\Delta$, without any loss of generality. However, it will prove helpful to be able to investigate various limits while holding various physical quantities such as $m$ or $\mu$ fixed, and this is done in the most transparent manner by keeping the definition of $\Delta$ completely independent of other quantities in the problem. Furthermore, to re-iterate the point we highlighted in $\S 1$, although it might seem more natural to specify $m^{2}$ and then seek the loading $\lambda$ as a function of $m$, some mathematical subtleties inherent in the description of the problem make this approach cumbersome. In particular, it is noted that the basic state satisfies equations (4) which depend on $\lambda$. Thus if we pursue the normal method of developing a series for $\lambda$ as a function of $m^{2}$ then there is the potential difficulty that as we proceed we need to keep careful track of the form of the basic state that may need to be reappraised in light of small changes to $\lambda$. To circumvent this inconvenience we instead decide to determine $m^{2}:=m^{2}(\lambda)$. There is no formal difficulty in adopting this viewpoint and nothing is lost so doing for once answers are determined it is elementary to invert our results and thereby express $\lambda:=\lambda(m)$ if preferred. At this stage there is one parameter yet to be fixed being the salient regime for the in-plane stretching $\mu$, but the relevant sizing becomes evident in the course of the calculations described below.

In the following we shall see that as we increase the magnitude of the dimensionless background tension $\mu$ the solution structures evolves through three distinct stages IIII. Each of these is somewhat intricate and inevitably requires the introduction of some notational complexity. Rather than minimising this by repeating symbols from stage to stage, and thereby risking having some notation with multiple meanings in various parts of the paper, we have chosen to have unambiguous designations. This might initially seem overwhelming, but the three structures that are developed in $\S 4, \S 5$ and $\S 6$ are separate of each other and each section can be treated as largely self-contained. In this way, the need to undertake extensive cross-referencing between the three calculations is hopefully mitigated as far as we are able.

3. The solution structure for $\Delta \gg 1$ : stage I. Given the form of (11), simple scaling arguments applied to the base-state equations (4) suggest that across the majority of circular plate, where $\rho=\mathcal{O}(1)$, we have

$$
\Theta=\Delta^{4 / 3} \Theta_{0}+\Delta^{-4 / 3} \Theta_{1} \ldots, \quad \Phi=\Delta^{8 / 3} \Phi_{0}+\Phi_{1}+\ldots
$$

Leading-order terms in (4) reduce to

$$
\Theta_{0} \Phi_{0}=-\lambda_{0} \rho^{2}, \quad \mathcal{L}_{0}^{(1)}\left[\Phi_{0}\right]=-\frac{\Theta_{0}^{2}}{2 \rho},
$$


from which it quickly follows that

$$
\mathcal{L}_{0}^{(1)}\left[\Phi_{0}\right]=-\frac{\lambda_{0}^{2} \rho^{3}}{2 \Phi_{0}^{2}}
$$

It is a routine exercise to show that at the centre of the plate $\Phi_{0} \sim A \rho+\mathcal{O}\left(\rho^{3}\right)$ for some constant $A \in \mathbb{R}$ that could be determined numerically, but whose value is immaterial for our immediate purposes. Rather, what is of more significance is the nature of the solution of (14) at the $\operatorname{rim} \rho \rightarrow 1^{-}$. In view of the boundary conditions (6) on the base state at $\rho=1$ we anticipate that, if $\mu$ is small, then $\Phi_{0} \rightarrow 0$ as $\rho \rightarrow 1^{-}$, which requires

(16) $\Phi_{0} \sim \delta\left(x^{2 / 3}+\ldots\right), \quad \Theta_{0} \sim-\frac{\lambda_{0}}{\delta} x^{-2 / 3}(1-\ldots), \quad$ where $\quad \delta \equiv\left(\frac{3}{2} \lambda_{0}\right)^{2 / 3}$

as $x \equiv 1-\rho \rightarrow 0^{+}$. (This expression follows immediately from enforcing the balance between the second derivative on the left hand side of (15) with the nonlinear term on the right hand side.)

This then highlights the significance of a suitable rim layer wherein the majority of the wrinkling will take place. Elementary scaling of the governing equations suggests that $x=\mathcal{O}\left(\Delta^{-1}\right)$, so we define

$$
\rho=1-\frac{X}{\Delta}, \quad X=\mathcal{O}(1)
$$

whereupon, governed by the behaviours (16), we expect that

$$
(\Phi, \Theta)=\Delta^{2}\left(\phi_{0}, \theta_{0}\right)+\Delta^{4 / 3}\left(\phi_{1}, \theta_{1}\right)+\ldots .
$$

If a dash denotes differentiation with respect to $X$, then substitution in (4) shows that the zeroth-order terms satisfy

$$
\theta_{0}^{\prime \prime}=\lambda_{0}+\phi_{0} \theta_{0}, \quad \phi_{0}^{\prime \prime}=-\frac{1}{2} \theta_{0}^{2}
$$

and matching with the outer behaviour (16) demands that $\phi_{0} \sim \delta X^{2 / 3}$ and $\theta_{0} \sim$ $-\left(\lambda_{0} / \delta\right) X^{-2 / 3}$ as $X \rightarrow \infty$.

It is the rim condition $\Phi(1)=\mu^{2}$ from (6) that provides the clue for the appropriate scaling for $\mu$. If we put

$$
\mu=\Delta \mu_{0}, \quad \mu_{0}=\mathcal{O}(1)
$$

then we must have

$$
\theta_{0}(0)=0 \quad \text { and } \quad \phi_{0}(0)=\mu_{0}^{2} .
$$

Clearly, the value of $\mu_{0}>0$ plays a significant role in setting the leading-order form of the basic state within the rim region and thus, presumably, is important in setting the loading that generates wrinkle modes. Hence we now work with $\mu_{0}$ assumed fixed and given, and seek to determine the value of $\lambda_{0}(m)$ that marks the onset of buckling. 
3.1. The structure of the eigen-deformation. Given the form of the basic solution we are able to proceed to examine the perturbation equations. We can thereby identify the key scalings that ensure that quadratic terms drive perturbations that are confined to a thin zone within the $X=\mathcal{O}(1)$ region. Put another way, the basic state develops a rim layer and inside that, already thin, layer sit the wrinkle modes. It can be verified that this inner rim later is of relative extent $\mathcal{O}\left(\Delta^{-1 / 4}\right)$, and so the wrinkle exists at some location $X=X_{0}$ about which we define the rescaled variable $Y=\mathcal{O}(1)$,

$$
Y:=\Delta^{1 / 4}\left(X-X_{0}\right) \quad \text { or } \quad \rho=1-\frac{X_{0}}{\Delta}-\frac{Y}{\Delta^{5 / 4}} .
$$

There are now two issues to be settled: (i) what sets the value of the location $X_{0}$ and (ii) how is the disturbance confined to the vicinity of this point? We can begin to address these questions by expanding the rim layer quantities $\phi_{j}(X)$ as Taylor series taken about $X=X_{0}$. This shows that where $Y=\mathcal{O}(1)$ we have

$$
\begin{aligned}
\Phi=\Delta^{2}\left(\phi_{00}+\Delta^{-1 / 4} \phi_{01} Y+\frac{1}{2} \Delta^{-1 / 2} \phi_{02} Y^{2}\right. & \left.+\frac{1}{6} \Delta^{-3 / 4} \phi_{03} Y^{3}+\ldots\right) \\
& +\Delta^{4 / 3}\left(\phi_{10}+\Delta^{-1 / 4} \phi_{11} Y+\ldots\right)
\end{aligned}
$$

where the constants $\phi_{i j}$ denote the $j^{t h}$ derivative of $\phi_{i}(X)$ evaluated at $X=X_{0}$. Taking derivatives shows that

$$
\frac{d \Phi}{d \rho}=-\Delta^{3} \phi_{01}-\Delta^{11 / 4} \phi_{02} Y-\frac{1}{2} \Delta^{5 / 2} \phi_{03} Y^{2}+\ldots
$$

and we remark that expressions completely analogous to (23) and (24) hold for $\Theta$ and its derivative, with the $\phi_{i j}$ replaced by $\theta_{i j}$ which represents the $j^{\text {th }}$-order derivative of $\theta_{i}(X)$ evaluated at $X=X_{0}$. Notice that although the base state correction term $\phi_{1}$ enters both the expressions (18) and the Taylor series (23), it is not required for the results we derive below. Hence, for reasons of brevity, we do not discuss $\phi_{1}$ (and $\theta_{1}$ ) further here, though of course their presence would have to be properly accounted for if we were to delve deeper into later terms in our series solutions.

3.2. The bifurcation equations. Given these proposed structures, and with the squared mode number $m^{2}$ defined by (12), the scene is now set for determining the important equations. We look for a solution of (8) of the form

$$
(W, \Psi)=\left(W_{0}, \Psi_{0}\right)+\Delta^{-1 / 4}\left(W_{1}, \Psi_{1}\right)+\Delta^{-1 / 2}\left(W_{2}, \Psi_{2}\right)+\ldots,
$$

and remember that $\Phi$ and its derivative are given by (23) and (24). On substituting (25) into the original equations (8), collecting like powers of $\Delta$, and then setting to zero their corresponding coefficients results in a hierarchy of coupled equations, as explained below.

Terms of $\mathcal{O}\left(\Delta^{6}\right)$ in the two equations yield

$$
\begin{gathered}
\mathcal{R}_{1}\left[W_{0}, \Psi_{0}\right] \equiv\left(M_{0}-\phi_{01}\right) W_{0}-\theta_{01} \Psi_{0}=0 \\
\mathcal{R}_{2}\left[W_{0}, \Psi_{0}\right] \equiv \theta_{01} W_{0}+M_{0} \Psi_{0}=0 .
\end{gathered}
$$


The consistency of this linear homogeneous system in $W_{0}$ and $\Psi_{0}$ requires

$$
M_{0}\left(M_{0}-\phi_{01}\right)+\theta_{01}^{2}=0 .
$$

At $\mathcal{O}\left(\Delta^{23 / 4}\right)$ it follows that

$$
\begin{gathered}
\mathcal{R}_{1}\left[W_{1}, \Phi_{1}\right]=Y \theta_{02} \Psi_{0}-\left(M_{1}-\phi_{02} Y\right) W_{0}, \\
\mathcal{R}_{2}\left[W_{1}, \Phi_{1}\right]=-\theta_{02} Y W_{0}-M_{1} \Psi_{0} .
\end{gathered}
$$

Again, a solution is only possible if suitable consistency conditions hold. The pair of equations (26) imply that $M_{0} \mathcal{R}_{1}(W, \Phi)+\theta_{01} \mathcal{R}_{2}(W, \Phi) \equiv 0$ as these two operators are linearly related. It follows that the system (28) is compatible only if

$$
M_{1}=0 \quad \text { and } \quad M_{0} \phi_{02}=2 \theta_{01} \theta_{02} .
$$

We need to proceed as far as $\mathcal{O}\left(\Delta^{11 / 2}\right)$. We determine that

$$
\begin{gathered}
\mathcal{R}_{1}\left[W_{2}, \Phi_{2}\right]=\theta_{02} Y \Psi_{1}+\phi_{02} Y W_{1}+\frac{1}{2} \theta_{03} Y^{2} \Psi_{0}-\left(M_{2}-\frac{1}{2} \phi_{03} Y^{2}\right) W_{0}+2 \frac{d^{2} W_{0}}{d Y^{2}}, \\
\mathcal{R}_{2}\left[W_{2}, \Phi_{2}\right]=-\theta_{02} Y W_{1}-\frac{1}{2} \theta_{03} Y^{2} W_{0}-M_{2} \Psi_{0}+2 \frac{d^{2} \Psi_{0}}{d Y^{2}} .
\end{gathered}
$$

The consistency of this pair requires

$$
\frac{d^{2} W_{0}}{d Y^{2}}+\left[\frac{M_{0} \phi_{03}-2 \theta_{01} \theta_{03}-2 \theta_{02}^{2}}{4\left(2 M_{0}-\phi_{01}\right)}\right] Y^{2} W_{0}-\frac{1}{2} M_{2} W_{0}=0,
$$

which, when cast in the generic form

$$
\frac{d^{2} W_{0}}{d Y^{2}}-\gamma Y^{2} W_{0}+\delta W_{0}=0,
$$

admits the exact solution $W_{0} \propto \exp \left(-\gamma^{1 / 2} Y^{2} / 2\right)$ if $\delta=\gamma^{1 / 2}$. This gives

$$
M_{2}=-2\left[\frac{2 \theta_{02}^{2}+2 \theta_{01} \theta_{03}-M_{0} \phi_{03}}{4\left(2 M_{0}-\phi_{01}\right)}\right]^{1 / 2},
$$

as long as $\gamma>0$. The expression $W_{0}(Y) \propto \exp \left(-\gamma^{1 / 2} Y^{2} / 2\right)$ proves that the solution is effectively confined to the $Y=\mathcal{O}(1)$ region subsumed within the $X=\mathcal{O}(1)$ rim layer governing the base structure.

We now have the information we require to uncover the location of the wrinkles centred at $X=X_{0}$. For a given $\lambda_{0}$ the leading-order rim solution $\left(\phi_{0}, \theta_{0}\right)$ satisfies the coupled system (19) subject to (21) and the matching conditions $\phi_{0} \sim \delta X^{2 / 3}$ and $\theta_{0} \sim-\left(\lambda_{0} / \delta\right) X^{-2 / 3}$ as $X \rightarrow \infty$. Wrinkling occurs with a scaled square mode number $M_{0}$ and is located at $X=X_{0}$, where $M_{0}$ and $X_{0}$ are determined by solving the consistency equations (27) and (29). Solution of this problem requires some associated numerical work, as explained briefly in the next section.

4. Numerical solution of the stage-I equations. Our computational task requires that, given the scaled constant $\mu_{0}>0$, we need to determine the relationship between $\lambda_{0}$ and $M_{0}$. It turns out that considerable simplification can be achieved by some judicious scaling. If we define a new rim co-ordinate $\widehat{X} \geq 0$ according to

$$
\widehat{X}:=\lambda_{0}^{1 / 4} X,
$$


and write the base structure variables $\phi_{0}=: \lambda_{0}^{1 / 2} \widehat{\phi}_{0}$ and $\theta_{0}=: \lambda_{0}^{1 / 2} \widehat{\theta}_{0}$, then it follows that

$$
\widehat{\theta}_{0}^{\prime \prime}=1+\widehat{\phi}_{0} \widehat{\theta}_{0}, \quad \widehat{\phi}_{0}^{\prime \prime}=-\frac{1}{2} \widehat{\theta}_{0}^{2},
$$

subject to the constraints

$$
\widehat{\phi}_{0} \sim \alpha \widehat{X}^{2 / 3}+\ldots, \quad \widehat{\theta}_{0} \sim-\frac{1}{\alpha} \widehat{X}^{-2 / 3}+\ldots \quad \text { as } \widehat{X} \rightarrow \infty ; \quad \alpha \equiv\left(\frac{3}{2}\right)^{2 / 3}
$$

together with

$$
\widehat{\theta}_{0}(0)=0 \quad \text { and } \quad \widehat{\phi}_{0}(0)=\widehat{\Lambda} ;
$$

here, we have introduced the definition

$$
\widehat{\Lambda}:=\frac{\mu_{0}^{2}}{\lambda_{0}^{1 / 2}} .
$$

If furthermore, we put $M_{0}=: \lambda_{0}^{3 / 4} \widehat{M}_{0}$ and denote by $\widehat{\phi}_{0 j}$ and $\widehat{\theta}_{0 j}$ the $j^{\text {th }}$ derivatives of $\widehat{\phi}_{0}$ and $\widehat{\theta}_{0}$ evaluated at $\widehat{X}=\widehat{X}_{0}$, then the consistency conditions (27) and (29) become just

$$
\widehat{M}_{0}\left(\widehat{M}_{0}-\widehat{\phi}_{01}\right)+\widehat{\theta}_{01}^{2}=0 \quad \text { and } \quad \widehat{M}_{0} \widehat{\phi}_{02}=2 \widehat{\theta}_{01} \widehat{\theta}_{02} .
$$

By this device we have reduced by one the dimension of the parameter space over which solution is required. For each $\widehat{\Lambda}$ there is one pair of corresponding $\left(\widehat{M}_{0}, \widehat{X}_{0}\right)$ and we are faced with a three-point boundary-value problem comprising the fourth-order system $(35)-(37)$ subject to consistency conditions to be imposed at a point $\widehat{X}_{0}$ that is part of the solution. This computation was carried out using standard routines available in MATLAB.

Some representative solutions are shown in Figure 3; in the left panel is illustrated the dependence of $\widehat{M}_{0}$ on $\widehat{\Lambda}$, while the right panel indicates the corresponding form of the location $\widehat{X}_{0}$ within the rim region. We note that for no initial in-plane stretching, i.e. $\widehat{\Lambda}=0$, we have finite values $\widehat{M}_{0} \simeq 0.8721$ and $\widehat{X}_{0} \simeq 1.066$. As $\widehat{\Lambda}$ increases so initially $\widehat{X}_{0}$ grows, but this trend is soon reversed and both $\widehat{M}_{0}$ and $\widehat{X}_{0}$ drop steadily with $\widehat{\Lambda}$. This suggests that to account for stronger stretching $\mu_{0} \gg 1$ (and so $\widehat{\Lambda} \gg 1$ by definition (38)) some sort of new structure ought to come into play in an appropriate large- $\widehat{\Lambda}$ limit. To unravel the corresponding details the first step is therefore to examine the nature of the solution of (35)-(37) subject to (39) as $\widehat{\Lambda} \rightarrow \infty$.

4.1. The solution of (35)-(39) for large $\widehat{\Lambda}$. Consideration of the boundary condition imposed on $\widehat{\phi}_{0}$ at $\widehat{X}=0$ together with the nature of the governing equations suggest that when $\widehat{\Lambda} \gg 1$ the solution develops a short-scale structure on a length $\mathcal{O}\left(\Lambda^{-1 / 2}\right)$. We therefore define

$$
\widehat{X}=\widehat{\Lambda}^{-1 / 2} z,
$$

and propose that the solution takes the form
(41) $\widehat{\theta}_{0}=\widehat{\Lambda}^{-1} \widetilde{\theta}_{0}(z)+\widehat{\Lambda}^{-3} \widetilde{\theta}_{1}(z)+\ldots$,
$\widehat{\phi}_{0}=\widehat{\Lambda} \widetilde{\phi}_{0}(z)+\widehat{\Lambda}^{-1} \widetilde{\phi}_{1}(z)+\widehat{\Lambda}^{-3} \widetilde{\phi}_{2}(z)+\ldots$ 

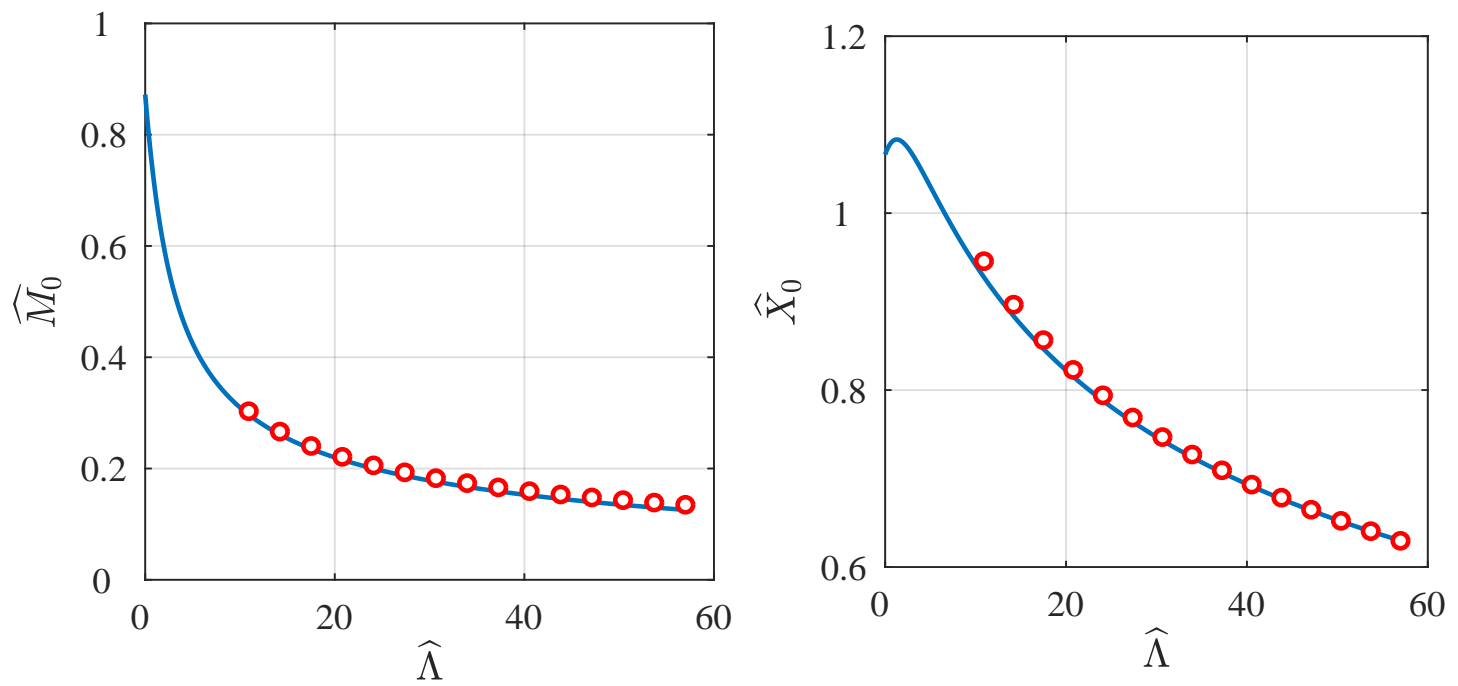

FIG. 3. The forms of $\widehat{M}_{0}$ (left) and $\widehat{X}_{0}$ (right) as functions of the parameter $\widehat{\Lambda}$ as determined from the solution of system (35)-(37) subject to (39) being satisfied at $\widehat{X}=\widehat{X}_{0}$. Shown as superimposed markers are the corresponding large- $\widehat{\Lambda}$ asymptotic results (46).

On substituting these forms in (35), comparison of like coefficients of $\widehat{\Lambda}$ in the two equations yield that

$$
\frac{d^{2} \widetilde{\theta}_{0}}{d z^{2}}=1+\widetilde{\theta}_{0} \widetilde{\phi}_{0}, \quad \frac{d^{2} \widetilde{\theta}_{1}}{d z^{2}}=\widetilde{\theta}_{0} \widetilde{\phi}_{1}+\widetilde{\theta}_{1} \widetilde{\phi}_{0}, \quad \frac{d^{2} \widetilde{\phi}_{0}}{d z^{2}}=\frac{d^{2} \widetilde{\phi}_{1}}{d z^{2}}=0 \quad \text { and } \quad \frac{d^{2} \widetilde{\phi}_{2}}{d z^{2}}=-\frac{1}{2} \widetilde{\theta}_{0}^{2} .
$$

In view of the boundary conditions we suppose that $\widetilde{\phi}_{0} \equiv 1$, a claim that can be checked later. Given this, it follows quickly that $\widetilde{\theta}_{0}=-1+\exp (-z)$ and we can also deduce that $\widetilde{\phi}_{1}$ is proportional to $z$. We cannot tie down this solution completely without recourse to the far-field conditions (36) for $\widehat{X} \rightarrow \infty$. The fact that the solution does not match directly onto the far-field requirements suggests strongly that the inner-solution zone must be supplemented by some form of outer structure. It is not difficult to verify that this outer zone lies where $\widehat{X}=\widehat{\Lambda}^{3 / 2} \widetilde{Y}$ with $\widetilde{Y}=\mathcal{O}(1)$ and that here

$$
\widehat{\theta}_{0}=\widehat{\Lambda}^{-1} \widetilde{\Theta}_{0}(\widetilde{Y})+\ldots, \quad \widehat{\phi}_{0}=\widehat{\Lambda} \widetilde{\Phi}_{0}(\widetilde{Y})+\ldots
$$

To match with the inner region requires that $\widetilde{\Theta}_{0} \rightarrow-1$ and $\widetilde{\Phi}_{0} \rightarrow 1$ as $\widetilde{Y} \rightarrow 0$. Leading-order terms in the governing equations (35) give

$$
\widetilde{\Theta}_{0} \widetilde{\Phi}_{0}+1=0 \quad \text { and } \quad \frac{d^{2} \widetilde{\Phi}_{0}}{d \widetilde{Y}^{2}}=-\frac{1}{2} \widetilde{\Theta}_{0}^{2} \quad \Longrightarrow \quad \frac{d^{2} \widetilde{\Phi}_{0}}{d \widetilde{Y}^{2}}=-\frac{1}{2 \widetilde{\Phi}_{0}^{2}} .
$$

This latter equation admits the exact solution $\widetilde{\Phi}_{0}=(1+3 \tilde{Y} / 2)^{2 / 3}$ which hence yields that $\widetilde{\Theta}_{0}=-(1+3 \widetilde{Y} / 2)^{-2 / 3}$. These expressions match automatically with the far-field requirements $(36)$ and with the inner-zone solutions as $\widetilde{Y} \rightarrow 0$. Furthermore we can now deduce that $\widetilde{\phi}_{1}=z$, rather than just being proportional to it, and it is then a 
routine matter to solve for $\widetilde{\phi}_{2}$. Taken together, this means that within the inner zone (40)

$$
\begin{aligned}
& \widehat{\theta}_{0}=\widehat{\Lambda}^{-1}[-1+\exp (-z)]+\ldots, \\
& \widehat{\phi}_{0}=\widehat{\Lambda}+\widehat{\Lambda}^{-1} z+\widehat{\Lambda}^{-3}\left[-\frac{1}{4} z^{2}+\exp (-z)-\frac{1}{8} \exp (-2 z)+\widetilde{c} z-\frac{7}{8}\right]+\ldots,
\end{aligned}
$$

where the precise value of $\widetilde{c} \in \mathbb{R}$ will not be required.

We have now shown that when $\widehat{\Lambda} \gg 1$ the appropriate solution of (35)-(37) develops a two-layer structure with an inner $\mathcal{O}\left(\widehat{\Lambda}^{-1 / 2}\right)$-zone and a wider outer region. We still need to identify the corresponding values of $\widehat{M}_{0}$ and $\widehat{X}_{0}$ that together fulfil the consistency requirements (39). The numerical solutions sketched in Figure 3 suggested that as $\widehat{\Lambda} \rightarrow \infty$ so $\widehat{X}_{0} \rightarrow 0$, and therefore it is unlikely that the consistency conditions will hold somewhere in the outer zone. Granted this, suppose that (39) apply at some point $\widehat{X}_{0}=\widehat{\Lambda}^{-1 / 2} z_{0}$ for some $z_{0}>0$ to be found. In order to satisfy (39) it is clear that the values of the various derivatives $\widehat{\theta}_{01}, \widehat{\theta}_{02}, \widehat{\phi}_{01}$ and $\widehat{\phi}_{02}$ need to be found. These can be inferred directly from our foregoing results (43), which yield

$$
\begin{array}{ll}
\widehat{\phi}_{01}=\widehat{\Lambda}^{-1 / 2}+\ldots, & \widehat{\theta}_{01}=-\widehat{\Lambda}^{-1 / 2} \exp \left(-z_{0}\right)+\ldots, \\
\widehat{\phi}_{02}=-\frac{1}{2} \widehat{\Lambda}^{-2}\left(1-\exp \left(-z_{0}\right)\right)^{2}+\ldots, & \widehat{\theta}_{02}=\exp \left(-z_{0}\right)+\ldots
\end{array}
$$

We proceed by examining the first of the consistency conditions in (39). Given the values noted in (44) there appear to be two possibilities: either $\widehat{M}_{0} \simeq-\widehat{\theta}_{01}^{2} / \widehat{\phi}_{01}$ or $\widehat{M}_{0} \simeq \widehat{\phi}_{01}=\Lambda^{-1 / 2}$. If we take the former option, routine algebra shows that the second consistency requirement $\widehat{M}_{0} \widehat{\phi}_{02}=2 \widehat{\theta}_{01} \widehat{\theta}_{02}$ cannot be satisfied. We are then left to conclude that

$$
\widehat{M}_{0} \simeq \widehat{\Lambda}^{-1 / 2} \quad \text { and } \quad \widehat{X}_{0} \simeq \widehat{\Lambda}^{-1 / 2} \ln (2 \widehat{\Lambda}), \quad \text { as } \quad \widehat{\Lambda} \rightarrow \infty,
$$

where the value of $\widehat{X}_{0}$ follows from the second consistency condition. These large$\widehat{\Lambda}$ predictions are superimposed on the results shown in Figure 3 , and both show excellent agreement with the direct numerical simulations.

5. The emergence of structure for larger values of $\mu$ : stage II. In the preceding sections we have sought to explain the structure of wrinkling eigendeformations with high mode numbers $\left(m \sim \Delta^{3 / 2}\right)$ when the in-plane stretching parameter $\mu$ is of size $\mathcal{O}(\Delta)$ : recall (12) and (20). Equation (11) reminds us that the corresponding loading for wrinkling is $\mathcal{O}\left(\Delta^{4}\right)$ and we now investigate how the situation needs to be modified as $\mu$ grows. The workings of the previous section show what is likely to happen as $\widehat{\Lambda}$ increases. In particular we observe that the square of the mode number $m^{2} \sim \Delta^{3} M_{0}=\Delta^{3} \lambda_{0}^{3 / 4} \widehat{M}_{0}$, and for $\widehat{\Lambda} \gg 1$ we predicted $\widehat{M}_{0} \sim \widehat{\Lambda}^{-1 / 2}=\lambda_{0}^{1 / 4} / \mu_{0}$ by definition (38). Hence the wrinkle mode number is

$$
m \sim \Delta^{3 / 2} \lambda_{0}^{1 / 2} / \mu_{0}^{1 / 2}
$$

We need to be careful that we continue to examine eigenstates with mode numbers consistent with those appropriate to stage I; that is, we should keep $m \sim \Delta^{3 / 2}$. This then suggests $\lambda_{0} \sim \mu_{0}$ while a second constraint for fixing the appropriate sizings for 
$\lambda_{0}$ and $\mu_{0}$ follows from the wrinkling structure itself. Using equation (32) and the scalings of $\S 3$, it follows that the wrinkling layer is of extent $\mathcal{O}\left(\Delta^{-5 / 4} \widehat{\gamma}^{-1 / 4}\right)$, where

$$
\widehat{\gamma} \equiv \lambda_{0}^{5 / 4}\left[\frac{-\widehat{M}_{0} \widehat{\phi}_{03}+2 \widehat{\theta}_{01} \widehat{\theta}_{03}+2 \widehat{\theta}_{02}^{2}}{4\left(2 \widehat{M}_{0}-\widehat{\phi}_{01}\right)}\right] .
$$

Given the asymptotic results (44), it transpires that the depth of the wrinkle zone is comparable to the distance of its centre from the rim when

$$
\Delta^{-5 / 4} \lambda_{0}^{-1 / 4} \mu_{0}^{-1 / 4} \sim \Delta^{-1} \quad \text { or } \quad \lambda_{0} \mu_{0} \sim \Delta .
$$

Taken with our earlier expectation $\lambda_{0} \sim \mu_{0}$ it is now clear that some new structure is anticipated once $\mu \equiv \Delta \mu_{0}$ becomes $\mathcal{O}\left(\Delta^{3 / 2}\right)$. To avoid introducing a plethora of new variables we recycle much of the preceding notation, changing only those parts that are crucial to avoid confusion.

Our discussion immediately above suggests that the stretching and loading must be scaled according to

$$
\mu=\Delta^{3 / 2} \mu_{0}^{\dagger}, \quad \lambda=\Delta^{9 / 2} \lambda_{0}^{\dagger}, \quad \text { for some } \mu_{0}^{\dagger}, \lambda_{0}^{\dagger}=\mathcal{O}(1),
$$

which replace (20) and (11), respectively. We have been careful to ensure that we continue to seek eigen-deformations with mode numbers $\mathcal{O}\left(\Delta^{3 / 2}\right)$, so suppose that

$$
m^{2}=M_{0}^{\dagger} \Delta^{3}+M_{1}^{\dagger} \Delta^{2}+\ldots .
$$

Once again, we proceed assuming that $\mu_{0}^{\dagger}$ is fixed and given, and endeavour to find the form of $\lambda_{0}^{\dagger}=\lambda_{0}^{\dagger}\left(M_{0}^{\dagger}\right)$ that marks the onset of buckling. Our previous asymptotics predict that the wrinkling is confined to an $\mathcal{O}\left(\Delta^{-1}\right)$-distance off the rim, so we can simply retain definition (17) with $\rho=1-X \Delta^{-1}$.

In view of the increase in the loading $\lambda$ the basic state is modified, though the key equations are only slightly altered. The basic state across the majority of the plate now satisfies

$$
\Theta=\Delta^{3 / 2} \Theta_{0}+\ldots, \quad \Phi=\Delta^{3} \Phi_{0}+\ldots,
$$

where

$$
\Theta_{0} \Phi_{0}=-\lambda_{0}^{\dagger} \rho^{2} \quad \text { and } \quad \mathcal{L}_{0}^{(1)}\left[\Phi_{0}\right]=-\frac{\left(\lambda_{0}^{\dagger}\right)^{2} \rho^{3}}{2 \Phi_{0}^{2}} .
$$

Previously we needed to solve for $\Phi_{0}$ subject to the requirement that it vanished as $\rho \rightarrow 1^{-}$; however, now the enhanced value of $\mu$ in (49) means that we simply require that $\Phi_{0}(1)=\left(\mu_{0}^{\dagger}\right)^{2}$. If we write $\Phi_{0} \equiv\left(\mu_{0}^{\dagger}\right)^{2} \phi_{0}$ then it follows that

$$
\mathcal{L}_{0}^{(1)}\left[\phi_{0}\right]=-\frac{\Gamma^{2} \rho^{3}}{2\left(\phi_{0}\right)^{2}} ; \quad \phi_{0}(0)=0, \quad \phi_{0}(1)=1 ; \quad \Gamma \equiv \frac{\lambda_{0}^{\dagger}}{\left(\mu_{0}^{\dagger}\right)^{3}} .
$$

We need to ascertain the behaviour of this solution in the rim zone $X=\mathcal{O}(1)$ and it is straightforward to deduce that if $\phi_{0}^{\prime}(1) \equiv \beta$ then in the rim zone

$$
\Phi=\Delta^{3}\left(\mu_{0}^{\dagger}\right)^{2}\left[1-\frac{\beta X}{\Delta}+\frac{1}{2}\left(1-\beta-\frac{1}{2} \Gamma^{2}\right) \frac{X^{2}}{\Delta^{2}}+\ldots\right] ; \quad \Theta=\mathcal{O}\left(\Delta^{3 / 2}\right) .
$$


If where $X=\mathcal{O}(1)$ the wrinkle adopts the form

$$
(W, \Psi)=\left(W_{0}^{\dagger}, \Psi_{0}^{\dagger}\right)+\Delta^{-1}\left(W_{1}^{\dagger}, \Psi_{1}^{\dagger}\right)+\ldots,
$$

then leading-order terms arising from substitution in (8) tell us that

$$
M_{0}^{\dagger}+\beta\left(\mu_{0}^{\dagger}\right)^{2}=0 \quad \text { and } \quad\left(\mu_{0}^{\dagger}\right)^{2} M_{0}^{\dagger} \Psi_{0}^{\dagger}=\lambda_{0}^{\dagger}(\beta-2) W_{0}^{\dagger} .
$$

At next order in equation (8a) we find that

$$
\left[2+\frac{\left(\mu_{0}^{\dagger}\right)^{2}}{M_{0}^{\dagger}}\right] \frac{d^{2} W_{0}^{\dagger}}{d X^{2}}-\left[\left(\mu_{0}^{\dagger}\right)^{2}\left(\beta+\frac{1}{2} \Gamma^{2}-1\right) X+M_{1}^{\dagger}\right] W_{0}^{\dagger}=0
$$

This equation is merely a scaled form of the ubiquitous Airy equation $y^{\prime \prime}-x y=0$, which is known to admit a solution with $y\left(x_{0}\right)=0$ and $y \rightarrow 0$ as $x \rightarrow \infty$ if $x_{0} \simeq$ -2.331 . Given this, we deduce that equation (57) enables $W_{0}^{\dagger} \rightarrow 0$ both as $X \rightarrow 0$ and as $X \rightarrow \infty$ if

$$
\text { (58) } \quad M_{1}^{\dagger} \simeq-2.331\left(M_{0}^{\dagger}\right)^{-1 / 3}\left[\left(\beta+\frac{1}{2} \Gamma^{2}-1\right)\left(\mu_{0}^{\dagger}\right)^{2}+2 M_{0}^{\dagger}\right]^{2 / 3}\left[2 M_{0}^{\dagger}+\left(\mu_{0}^{\dagger}\right)^{2}\right]^{1 / 3} \text {. }
$$

We now have the elements required to determine the loading parameter $\lambda_{0}^{\dagger}$ in terms of $\mu_{0}^{\dagger}$. The key to unlocking this dependence lies in the requirement $\phi_{0}^{\prime}(1) \equiv \beta$ and the consistency condition (56a) combined with the basic state equation (53). This second-order equation already is subject to the two requirements, $\phi_{0}(0)=0$ and $\phi_{0}(1)=1$, and the third constraint $\phi_{0}^{\prime}(1)=-M_{0}^{\dagger} /\left(\mu_{0}^{\dagger}\right)^{2}$, which follows directly from $\phi_{0}^{\prime}(1) \equiv \beta$ and (56a), means that a solution only exists for certain values of $\Gamma$. We can write this in the alternative form

$$
\lambda_{0}^{\dagger}=\left(\mu_{0}^{\dagger}\right)^{3} G\left[\frac{M_{0}^{\dagger}}{\left(\mu_{0}^{\dagger}\right)^{2}}\right]
$$

for some function $G[\cdot]$ that can only be determined numerically; the form of this function is illustrated in Figure 4.

It is a straightforward computational exercise to show that problem (53) admits a solution with $\phi_{0}^{\prime}(1)=0$ when $\Gamma=K_{0} \approx 3.212$. This then tells us that for large $\mu_{0}^{\dagger}$ (and small $\beta$ ) then $\lambda_{0}^{\dagger} \simeq K_{0}\left(\mu_{0}^{\dagger}\right)^{3}$. Moreover, if we look for a solution of (53) as a regular series in inverse powers of $\mu_{0}^{\dagger}$ we can derive the two-term result

$$
\lambda_{0}^{\dagger}=K_{0}\left(\mu_{0}^{\dagger}\right)^{3}+1.217 M_{0}^{\dagger} \mu_{0}^{\dagger}+\ldots,
$$

which is included on Figure 4. It is observed that agreement is excellent, even for surprisingly modest values of $\mu_{0}^{\dagger}$.

These features forecast the expected behaviours at even larger values of $\mu$. As $\mu_{0}^{\dagger}$ grows so the leading order loading parameter $\lambda_{0}^{\dagger}$ becomes independent of the mode number $M_{0}^{\dagger}$, and the fact that the quantity $M_{1}^{\dagger} \sim \mathcal{O}\left(\left(\mu_{0}^{\dagger}\right)^{2}\right)$, according to (58), means that a restructuring should be anticipated once $\mu_{0}^{\dagger}=\mathcal{O}\left(\Delta^{1 / 2}\right)$. Then $\mu=\mathcal{O}\left(\Delta^{2}\right)$ and this last stage is described next. 


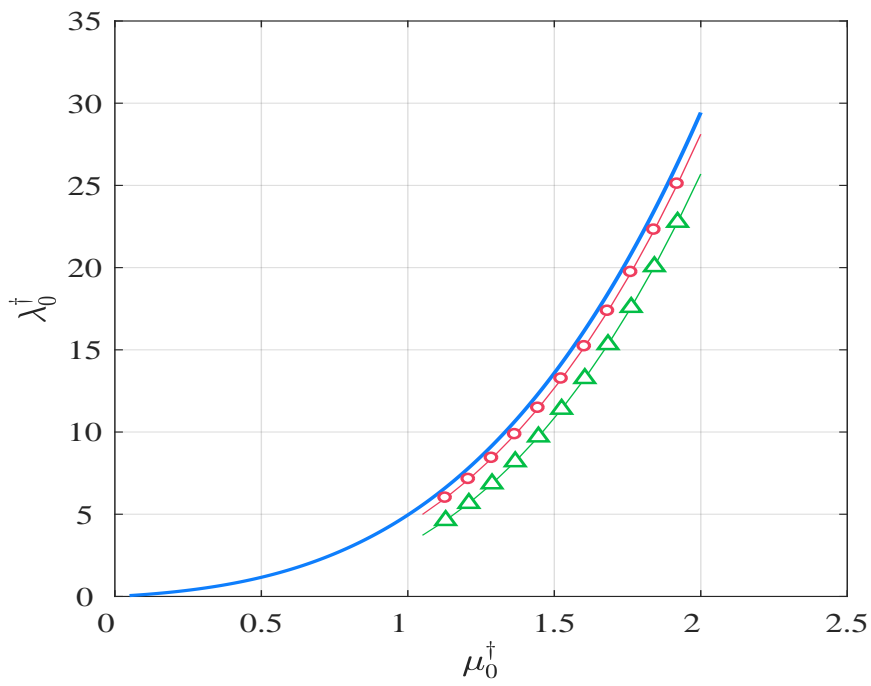

FIG. 4. The dependence of $\lambda_{0}^{\dagger}$ on $\mu_{0}^{\dagger}$ according to (59) when $M_{0}^{\dagger}=1$. Shown superimposed on this plot are the one- and two-term asymptotic results (60), which correspond to the triangular and round markers, respectively.

6. Stage III: strong stretching. Guided by the previous analysis we can quickly sketch the structure appropriate when

$$
\mu=\Delta^{2} \widetilde{\mu}, \quad \text { with } \quad \widetilde{\mu}=\mathcal{O}(1) .
$$

We anticipate that once more $m^{2}=\Delta^{3} \widetilde{M}$, for some $\widetilde{M}=\mathcal{O}(1)$, but that the commensurate loading is now

$$
\lambda=K_{0} \widetilde{\mu}^{3} \Delta^{6}+\widetilde{\lambda}_{1} \Delta^{5}+\widetilde{\lambda}_{2} \Delta^{4}+\ldots,
$$

where $\lambda_{j}=\mathcal{O}(1)(j=1,2)$ are yet to be determined. In passing we remark that this scaling that $m \sim \mu^{3 / 4}$ was first derived using asymptotic arguments by Coman \& Bassom [8] and later Davidovitch et al. [20] gave a simple argument based on scaling to confirm this result. Earlier in the paper we stressed our desire to take a solitary one-term form for $\lambda$ and, at face value, it seems that we are now deliberately deviating from this route. The reason is not difficult to appreciate; at such high values of $\mu$ the first term in the loading form (62) is independent of the wrinkle mode number $m$ according to the predictions of stage II. Thus, a simple one-term form for $\lambda$ would no longer be adequate to capture any wavenumber variation whatsoever, which forces our consideration of the more complicated (62). Now, across the main part of the plate, the series (51) becomes

$$
\Phi=\Delta^{4} \Phi_{0}+\Delta^{3} \Phi_{1}+\Delta^{2} \Phi_{2}+\ldots \quad \text { and } \quad \Theta=\Delta^{2} \Theta_{0}+\Delta \Theta_{1}+\Theta_{2}+\ldots,
$$

where $\Phi_{j} \equiv \Phi_{j}(X)$ and $\Theta_{j} \equiv \Theta_{j}(X)(j=0,1,2, \ldots)$ are to be determined. Note that if we write $\Phi_{0}=\widetilde{\mu}^{2} \phi_{0}$, then $\phi_{0}$ satisfies the equation (53) with the value $\Gamma=K_{0}$, by virtue of which we are guaranteed that $\Phi_{0}^{\prime}(1)=0$. Thus, we are able to express the form of the base state in the $X=\mathcal{O}(1)$ rim region to obtain the counterpart to (54) 
in the form

$$
\Phi=\widetilde{\mu}^{2} \Delta^{4}+\left(A_{22} X^{2}+A_{21} X\right) \Delta^{2}+\left(A_{13} X^{3}+A_{12} X^{2}+A_{11} X\right) \Delta+\ldots
$$

$$
\Theta=B_{20} \Delta^{2}+\left(B_{11} X+B_{10}\right) \Delta+\ldots
$$

where

$$
\begin{aligned}
& A_{22}:=\frac{1}{2}\left(1-\frac{1}{2} K_{0}^{2}\right) \widetilde{\mu}^{2}, \quad A_{13}:=\frac{1}{6}\left(3+K_{0}^{2}\right) \widetilde{\mu}^{2}, \quad B_{20}:=-K_{0} \widetilde{\mu}^{3}, \\
& A_{21}:=\frac{\widetilde{\lambda}_{1} K_{1}}{\widetilde{\mu}}, \quad A_{12}:=-\frac{\widetilde{\lambda}_{1}}{2 \widetilde{\mu}}\left(K_{0}-K_{1}\right), \quad B_{11}:=2 K_{0} \widetilde{\mu}^{3}, \\
& A_{11}:=\frac{\widetilde{\lambda}_{2} K_{1}}{\widetilde{\mu}}+\frac{\widetilde{\lambda}_{1}^{2} K_{2}}{\widetilde{\mu}^{4}}, \quad B_{10}:=-\widetilde{\lambda}_{1} .
\end{aligned}
$$

Standard numerical work (which is relegated to the supplementary material) shows that $K_{0} \simeq 3.212, K_{1} \simeq 0.5179$ and $K_{2} \simeq 0.0389$. In the expression (63a) we note the absence of the $\Delta^{3}$ term which is a direct consequence of the fact that $\Phi_{0}^{\prime}(1)=0$. We can use the approximation of the basic state (63) to capture the asymptotic structure of the wrinkles. To this end we shall employ the ansatz

$$
W=\widetilde{W}_{0}+\widetilde{W}_{1} \Delta^{-1}+\ldots \quad \text { and } \quad \Psi=\widetilde{\Psi}_{0} \Delta^{-1}+\widetilde{\Psi}_{1} \Delta^{-2}+\ldots
$$

The second equation in (8) gives an algebraic constraint, $\widetilde{M} \Psi_{0}+2 K_{0} \widetilde{\mu}^{3} W_{0}=0$; thus, the terms in the expansion (64b) are quite passive and respond to what the $W_{j}$ $(j=0,1, \ldots)$ components need to do. However, use of $(64)$ in $(8 \mathrm{a})$ yields

$$
\text { (65) } \frac{d^{2} \widetilde{W}_{0}}{d X^{2}}-(\alpha X-\beta) \widetilde{W}_{0}=0 \quad \text { with } \quad \alpha:=-\frac{2 \widetilde{M} A_{22}}{\widetilde{\mu}^{2}}, \quad \beta:=-\frac{\widetilde{M}\left(\widetilde{M}-A_{21}\right)}{\widetilde{\mu}^{2}} .
$$

We recognise this equation once again as related to an Airy form, and elementary algebra shows that a non-trivial solution with $\widetilde{W} \rightarrow 0$ as $X \rightarrow 0$ and $X \rightarrow \infty$ is possible if

$$
\widetilde{\lambda}_{1} K_{1}=\widetilde{\mu} \widetilde{M}+\xi_{0}\left(\frac{1}{2} K_{0}^{2}-1\right)^{2 / 3} \widetilde{\mu}^{5 / 3} \widetilde{M}^{-1 / 3}
$$

where $\operatorname{Ai}\left(-\xi_{0}\right)=0, \xi_{0} \simeq 2.331$. Now, while the leading-order term in (62) was independent of $\widetilde{M}$, we observe that $\widetilde{\lambda}_{1} \rightarrow \infty$ both as $\widetilde{M} \rightarrow 0$ and as $\widetilde{M} \rightarrow \infty$. Thus, we can identify the wavenumber that corresponds to the least loading, and minimizing $\widetilde{\lambda}_{1}$ with respect to $\widetilde{M}$ gives the critical point $\left(\widetilde{M}_{c}, \widetilde{\lambda}_{1 c}\right) \simeq(1.6877,13.0346)$.

We remark that the solution $\widetilde{W}_{0}$ does not fulfil all eight of the rim conditions (10) prescribed. This merely reflects the fact that the majority of the wrinkle zone is governed by a system of order less than eight, which means that not all the constraints can be satisfied. This does not present any problem and just points to the fact that the $\mathcal{O}\left(\Delta^{-1}\right)$ rim zone contains an inner region in which the aforementioned requirements can be ensured. The details of this inner zone affect later terms in our asymptotic series, in particular, they do influence the form of $\widetilde{\lambda}_{2}$ in (62). The manipulations required are routine but lengthy so, in the interest of brevity, the details 
of the corresponding analysis are consigned to the supplementary material. Here, we simply state the final results

$$
\text { (67) } \lambda_{c}=3.212 \mu^{3}+13.0346 \mu^{5 / 2}+54.8417 \mu^{2}+\ldots \quad \text { and } \quad m_{c}^{2}=1.6877 \mu^{3 / 2}+\ldots
$$

The predictions of these last formulae are illustrated in Figure 5, where we compare them with some direct numerical simulations of (8)-(10). It is clear that the agreement is very good. In particular, in the left window the relative errors range from $10 \%$ at $\mu=80$ to $5.7 \%$ when $\mu=120$ and are merely $2.8 \%$ once $\mu=200$. The predictions of the critical wavenumber differ from the simulations by about $5 \%$ when $\mu=180$; although these relative errors are slightly larger than for the critical loading values it should be remembered that the asymptotic result (67b) consists of only one term. Better improvement could be expected should further terms in (67b) be developed but this simple result is sufficiently accurate that the additional effort necessary to extricate higher order terms is arguably not commensurate with the likely marginal improvement in results.
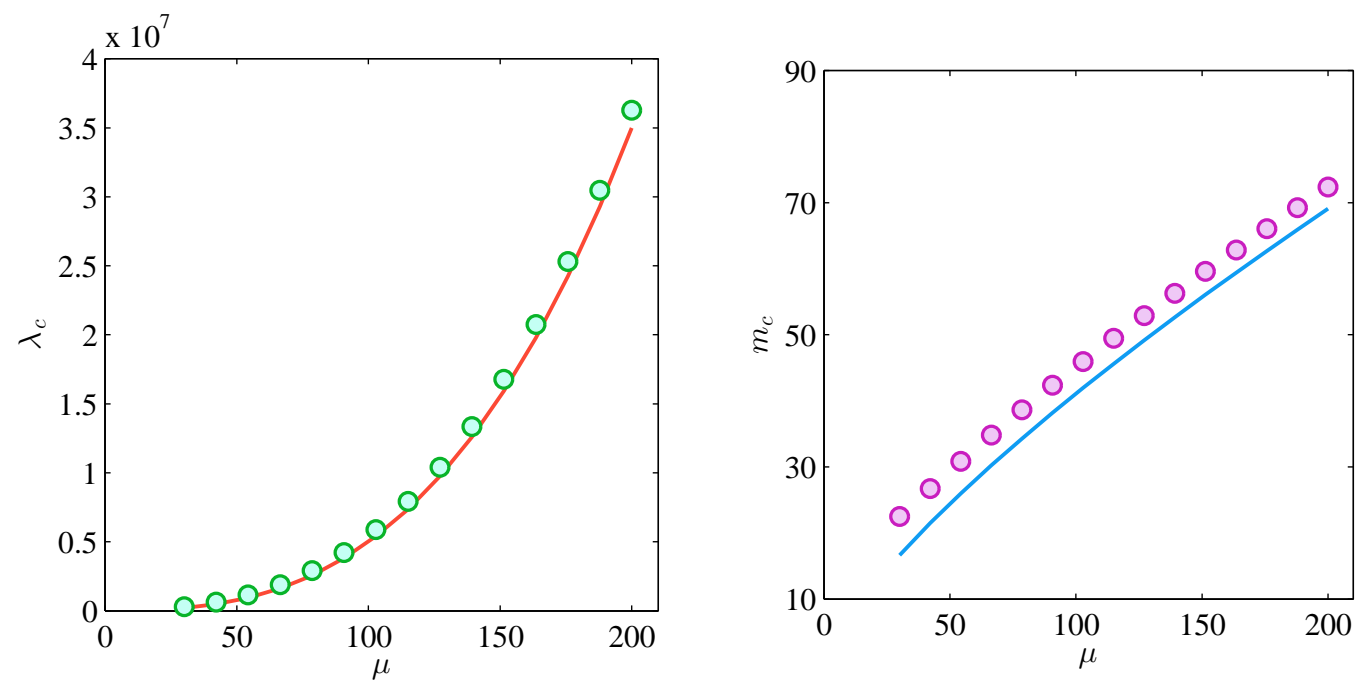

FIG. 5. Comparisons between direct numerical simulations of the boundary-value problem (8)-(10) and the critical values (67) for $30 \leq \mu \leq 200$. The markers correspond to the former set of data, while the continuous curves represent the asymptotic results.

7. Discussion. In this article we have endeavoured to provide a detailed description of the short-wavelength wrinkle modes that develop in a uniformly stretched weakly clamped circular plate subjected to a transverse pressure. Three distinct regimes of initial stretching have been identified (see Figure 6); in the first of these the eigenmodes are located off the rim of the plate at a location determined by the solution of a pair of consistency conditions. As the size of the stretching $\mu$ increases then the wrinkles effectively sit at the rim, where they are governed by the solution of a scaled Airy equation. A third regime is suggested in which the leading-order loading required for wrinkling loses all dependence on the mode number. 


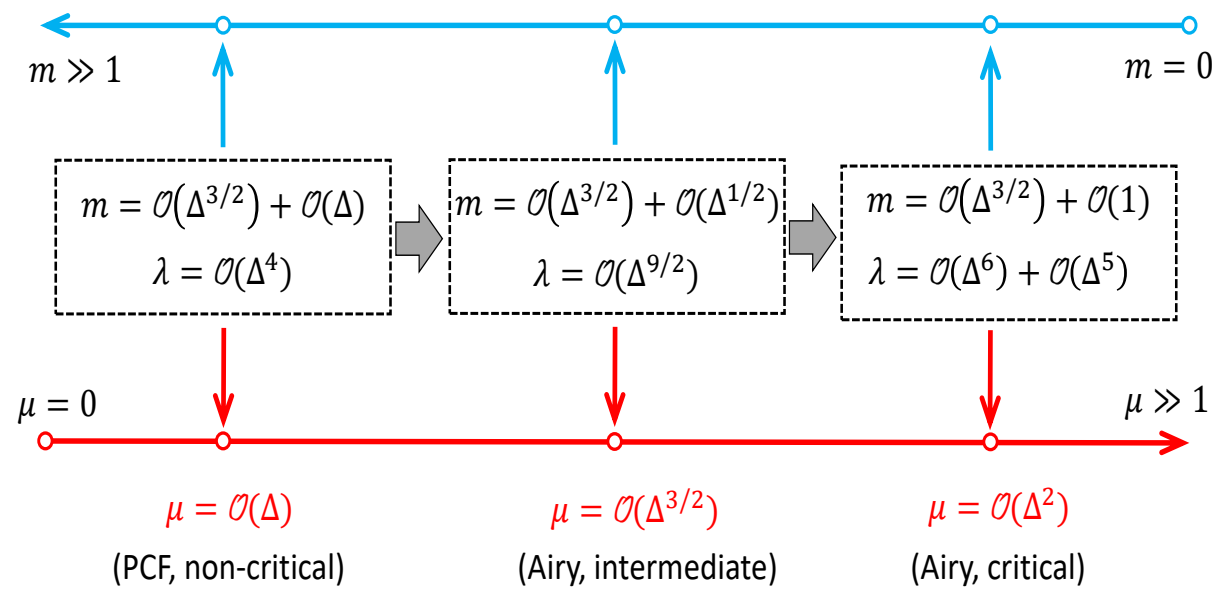

FIG. 6. Schematic of the asymptotic regimes studied. Upper line indicates the size of the correction to the leading order wavenumber $\mathcal{O}\left(\Delta^{3 / 2}\right)$.

At the outset our principal motivation behind this work was to shed light on the nature of the asymptotic decoupling of the FvK system found recently in some related studies $[5,6]$. Although there are a number of non-trivial examples in the literature in which the asymptotic decoupling of the FvK nonlinear equations has been encountered, for example [10]-[11], it should be emphasised that the nature of this phenomenon was actually quite different. Indeed, a close look indicates that the aforementioned references were concerned with out-of-plane bending perturbations from a state of plane stress. As a consequence, the compatibility relation in the FvK system decoupled at leading-order, giving rise to the standard linear bi-harmonic equation for the stress function, and this had the effect of turning the equilibrium equation into an expression solvable in closed form. So in spite of the fact that the analysis was ostensibly nonlinear, those works ended up dealing with a weak nonlinear perturbation from a linear plane-stress elastic state. By contrast, the situation present in our work is exactly the opposite. Here our perturbations take place relatively remote from the original flat state of the circular plate; exactly how remote is something that is controlled by the nonlinear basic state. This has significant ramifications for the subsequent asymptotic analysis as the nature of basic state is one of the critical elements in the implementation of our singular perturbation strategies.

It is important to appreciate some of the inherent limitations of our results. We have been exclusively focused on the onset of wrinkling which is acknowledged as being very awkward to observe in the laboratory. While there are numerous valid reasons for understanding onset (or near-threshold phenomena [20]), from the practical standpoint wrinkles well into the post-buckling regime are much easier to produce. In the far-post-buckling situation traditional simplified theories have been developed based on tension field theory [21], [22]. The approach taken by tension-field theory is in marked contrast to the bifurcation technique adopted here. Tension-field theory in some sense smears out the individual wrinkles and seeks to trace the evolution of the boundary separating the winkled and un-wrinkled areas. As further evidence that the post-buckling regime can behave very differently to the onset problem, we note recent results that suggest how spatially varying wavenumbers can be dramatically affected by increasing the load; see Paulsen et al. [23] and Taffetani \& Vella [24], to name just 
two studies of these effects.

It is helpful to note that our results need to be considered carefully if generalisations to other geometries are contemplated. An obvious question is to ask how our work may be applied to annular plates. In our present study the existence of the edge instability is contingent upon the presence of compressive stresses near the circumference which is guaranteed if the outer edge of the plate is weakly clamped or pinned. If there is also uniform stretching applied along the outer circumference then one has a handle on the extent of the region of compressive stresses and this is the role played by our parameter $\mu$. For an annular plate with a traction-free inner boundary, weakly clamped along the outer rim, and subjected to uniform stretching along that edge there will be no compressive stresses in the annulus according to the Lamé solution. If transverse pressure is also applied then the region of compressive stresses will be situated near the outer rim and this will be an entirely nonlinear phenomenon. Haughton \& McKay [25] have considered the plane-stress problem for an annular membrane in the case of a nonlinear Varga material and with several types of boundary conditions. The principal stresses were found to be always tensile if the inner boundary is stress free.

Our problem here has the feature that the loading intimately ties together the basic state with the infinitesimal wrinkle pattern. The usual approach taken in these types of problems is to determine the underlying basic state and then adjust the loading, which plays the role of an eigenvalue, so that non-trivial modes are possible. Here the situation is somewhat different. The value of $\lambda$ plays a pivotal role in the form of the basic state so that both this quantity and the perturbation structure really need to be developed in tandem. This is the feature that suggested it would be advantageous to view $\lambda$ as given and then calculate the associated wrinkle wavenumber. This strategy has enabled us to monitor the stability characteristics of the system as the in-plane loading varies from completely unstretched right through to a taut geometry. Whilst we have been able to implement similar techniques in related situations, we believe this is first example where it has proved possible to track the effect of a varying physical parameter over such an extended regime. It would be of considerable interest to know whether the problem we have here is somewhat special in that respect or whether the approach has more general applicability.

The referees are thanked for their numerous comments which led to improvements in the paper.

\section{REFERENCES}

[1] P.G. Ciarlet, Mathematical Elasticity (vol. 2: Theory of Plates), North-Holland, Amsterdam, 1997.

[2] B. Audoly, And Y. Pomeay, Elasticity and Geometry: from hair curls to the non-linear response of shells, Oxford University Press, Oxford, 2010.

[3] J.W. Hutchinson, And Z. Suo, Mixed mode cracking in layered materials, Adv. Appl. Mech., 29 (1991), pp. 63-93.

[4] C.D. Coman, AND A.P. BAssom, On the nonlinear membrane approximation and edgewrinkling, Int. J. Solids Struct., 82 (2016), pp. 85-94.

[5] C.D. Coman, And A.P. Bassom, Singularities and wrinkling: the case of a concentrated force, Int. J. Eng. Sci., 106 (2016), pp. 229-244.

[6] C.D. Coman, M.T. Matthews, And A.P. Bassom, Asymptotic phenomena in pressurised thin films, Proc. Roy. Soc. Lond. A, 471 (2015), Article number 20150471.

[7] C.D. Coman, Asymmetric bifurcations in a pressurised circular thin plate under initial tension, Mech. Res. Comm., 47 (2013), pp. 11-17.

[8] C.D. COMAn, AND A.P. BAssom, On the wrinkling of a pre-stressed annular thin film in 
tension, J. Mech. Phys. Solids, 55 (2007), pp. 1601-1617.

[9] C.D. COMAN, AND D.M. HAUGhton, Localized wrinkling instabilities in radially stretched annular thin films, Acta Mech., 55 (2006), pp. 179-200.

10] W.H. WitTrick, Axisymmetrical bending of a highly stretched annular plate, Quart. J. Mech. Appl. Math., 18 (1965), pp. 11-24.

[11] W.B. FRASER, Bending of a highly stretched elastic plate containing an eccentrically platereinforced circular hole, Int. J. Solids Structures, 11 (1975), pp. 501-518.

$12]$ G. Junkin, AND R. DAvis, General non-linear plate theory applied to a circular plate with large deflections. Int. J. Solids Struct., 9 (1972), pp. 84-93.

[13] J.G. Simmonds, And A. Libai, Asymptotic forms of a simplified version of the non-linear Reissner equations for clamped elastic spherical caps under outward pressure, Comp. Mech., 2 (1987) pp. 231-244.

[14] U. Komaragiri, M.R. Begley, and J.G. Simmonds, The mechanical response of freestanding circular elastic films under point and pressure loads, ASME, J. Appl. Mech., 72 (2005), pp. 203-212.

[15] V.L. Berdichevsky, Variational Principles of Continuum Mechanics, Nauka, Moscow, 1983.

[16] D.H. Hodges, Nonlinear Composite Beam Theory, American Institute of Aeronautics and Astronautics Inc.. Roston VA, 2006.

[17] M. TAylor, B. Davidovitch, Z. QiU, And K. Bertoldi, A comparative analysis of numerical approaches to the mechanics of elastic sheets, J. Mech. Phys. Solids, 79 (2015), pp. 92-107.

[18] G.G. AdAms, Elastic wrinkling of a tensioned circular plate using von-Kármán plate theory ASME J. Appl. Mech., 60 (1993), pp. 520-525.

[19] H. Troger, And A. Steinde, Nonlinear Stability and Bifurcation Theory, Springer-Verlag, Wien, 1991.

[20] B. Davidovitch, R.D. Schroll, D. Vella, M. Adda-Bedia, And E.A. Cerda, Prototypical model for tensional wrinkling in thin sheets, Proc. National Academy Sciences, 108 (2011), pp. $18227-18232$.

[21] A.C. PIPKIN, The relaxed energy density for isotropic elastic membranes, IMA J. appl. Math., 36 (1986), pp. 85-99.

[22] D.J. Steigmann, Tension-Field theory, Proc. R. Soc. Lond. A 429 (1990), pp. 141-173.

[23] J.D. Paulsen, E. Hohlfeld, H. King, J. Huang, Z. Qiu, T.P. Russell, N. Menon, D. VElla, AND B. DAVIDOVITCH, Curvature-induced stiffness and the spatial variation of wavelength in wrinkled sheets, Proc. National Academy Sciences, 113 (2016), pp. 11441149 .

[24] M. Taffetani, and D. Vella, Regimes of wrinkling in pressurized elastic shells, Phil. Trans Roy. Soc. Lond. A, 375 (2017). Article number 20160330.

[25] D.M. Haughton, AND A.B. MCKAY, Wrinkling of annular discs subjected to radial displacements, Int. J. Engng. Sci., 33 (1995), pp. 335-350. 


\title{
SUPPLEMENTARY MATERIALS: WRINKLING STRUCTURES AT \\ THE RIM OF AN INITIALLY STRETCHED CIRCULAR THIN PLATE SUBJECTED TO TRANSVERSE PRESSURE*
}

\author{
CIPRIAN D. COMAN ${ }^{\dagger}$ AND ANDREW P. BASSOM ${ }^{\ddagger}$
}

SM1. Background. For easy reference we start by listing below the main equations from [SM1]. The basic state is described by the main fields $\Theta \equiv \Theta(\rho)$ and $\Phi \equiv \Phi(\rho)$, which satisfy the nonlinear equations

$$
\frac{d^{2} \Theta}{d \rho^{2}}+\frac{1}{\rho} \frac{d \Theta}{d \rho}-\frac{\Theta}{\rho^{2}}=\lambda \rho+\frac{\Theta \Phi}{\rho} \quad \text { and } \quad \frac{d^{2} \Phi}{d \rho^{2}}+\frac{1}{\rho} \frac{d \Phi}{d \rho}-\frac{\Phi}{\rho^{2}}=-\frac{\Theta^{2}}{2 \rho},
$$

subject to the constraints

$$
\begin{array}{ll}
\Theta(1)=0, & \Phi(1)=\mu^{2}, \\
\Theta(0)=0, & \Phi(0)=0 .
\end{array}
$$

The incremental radial amplitudes $(W, \Psi)$ satisfy two coupled linear bifurcation equations,

$$
\mathcal{L}_{11}[W]+\mathcal{L}_{12}[\Psi]=0 \quad \text { and } \quad \mathcal{L}_{21}[W]+\mathcal{L}_{22}[\Psi]=0
$$

where

$$
\mathcal{L}_{11} \equiv\left[\mathcal{L}_{0}^{(m)}\right]^{2}-\frac{1}{\rho} \frac{d}{d \rho}\left(\Phi \frac{d}{d \rho}\right)+\frac{d \Phi}{d \rho}\left(\frac{m}{\rho}\right)^{2}, \quad \mathcal{L}_{22} \equiv\left[\mathcal{L}_{0}^{(m)}\right]^{2}
$$

$$
\mathcal{L}_{12}=-\mathcal{L}_{21} \equiv-\frac{1}{\rho} \frac{d}{d \rho}\left(\Theta \frac{d}{d \rho}\right)+\frac{d \Theta}{d \rho}\left(\frac{m}{\rho}\right)^{2}, \quad \mathcal{L}_{0}^{(k)} \equiv \frac{1}{\rho} \frac{d}{d \rho}\left(\rho \frac{d}{d \rho}\right)-\frac{k^{2}}{\rho^{2}} .
$$

The corresponding boundary conditions correspond to a weakly clamped plate and assume the form

$$
W=\frac{d W}{d \rho}=\Psi=\frac{d \Psi}{d \rho}=0, \quad \text { for } \quad \rho \in\{0,1\} .
$$

SM2. Basic state. Let us recall the main scalings from $\S 6$ in [SM1],

$$
\mu=\Delta^{2} \widetilde{\mu}, \quad \widetilde{\mu}=\mathcal{O}(1),
$$

and $m^{2}=\Delta^{3} \widetilde{M}$, for some $\widetilde{M}=\mathcal{O}(1)$; also, our loading can be expressed as

$$
\lambda=K_{0} \widetilde{\mu}^{3} \Delta^{6}+\widetilde{\lambda}_{1} \Delta^{5}+\widetilde{\lambda}_{2} \Delta^{4}+\ldots,
$$

for some $\tilde{\lambda}_{j}=\mathcal{O}(1)(j=1,2, \ldots)$. Our main goal is to find $\tilde{\lambda}_{1}$ and $\tilde{\lambda}_{2}$.

\footnotetext{
* Submitted to the editors January 18, 2018.

†School of Mathematical Sciences, University of Nottingham, University Park, Nottingham NG7 2RD, UK (cdc3p@yahoo.com)

$¥$ School of Physical Sciences, University of Tasmania, Private Bag 37, Hobart TAS 7001, Australia (andrew.bassom@utas.edu.au)
} 
A little more than a simple exercise in elementary algebra indicates that away from the rim of the plate $(\rho=1)$ our basic-state fields must be expanded acording to

$$
\Phi=\Delta^{4} \Phi_{0}+\Delta^{3} \Phi_{1}+\Delta^{2} \Phi_{2}+\ldots
$$

$$
\Theta=\Delta^{2} \Theta_{0}+\Delta \Theta_{1}+\Theta_{2}+\ldots
$$

where the behaviours of the unknown coefficient functions $\Theta_{j} \equiv \Theta_{j}(\rho)$ and $\Phi_{j} \equiv \Phi_{j}(\rho)$ $(j=0,1,2, \ldots)$ can be found as explained below.

Substituting (SM8) in (SM1a) leads to the algebraic relations

$$
\begin{aligned}
& -K_{0} \tilde{\mu}^{3} \rho^{2}=\Theta_{0} \Phi_{0}, \\
& -\widetilde{\lambda}_{1} \rho^{2}=\Theta_{0} \Phi_{1}+\Theta_{1} \Phi_{0}, \\
& -\widetilde{\lambda}_{2} \rho^{2}=\Theta_{0} \Phi_{2}+\Theta_{1} \Phi_{1}+\Theta_{2} \Phi_{0},
\end{aligned}
$$

while the other base equation, (SM1b), yields a sequence of differential equations

$$
\begin{aligned}
& \mathcal{L}_{0}^{(1)}\left[\Phi_{0}\right]=-\frac{\Theta_{0}^{2}}{2 \rho}, \\
& \mathcal{L}_{0}^{(1)}\left[\Phi_{1}\right]=-\frac{\Theta_{0} \Theta_{1}}{\rho}, \\
& \mathcal{L}_{0}^{(1)}\left[\Phi_{2}\right]=-\frac{\Theta_{1}^{2}+2 \Theta_{0} \Theta_{2}}{2 \rho} .
\end{aligned}
$$

SM2.1. Zeroth order. Eliminating $\Theta_{0}$ between (SM9a) and (SM10a), and further putting $\Phi_{0}=: \widetilde{\mu}^{2} \phi_{0}$, gives that

$$
\phi_{0}^{\prime \prime}+\frac{1}{\rho} \phi_{0}^{\prime}-\frac{1}{\rho^{2}} \phi_{0}=-\frac{K_{0}^{2} \rho^{3}}{2 \phi_{0}^{2}}, \quad \phi_{0}(0)=0, \quad \phi_{0}(1)=1, \quad \phi_{0}^{\prime}(1)=0 .
$$

We recall that the base condition $\Phi=\mu^{2}$ on $\rho=1$ leads to the middle of the above boundary conditions, while the vanishing of the derivative at the rim was provoked by the outcome of stage II. By solving numerically the eigenvalue problem (SM11) we find

$$
K_{0} \simeq 3.212
$$

We are going to need the form of the basic state inside the rim zone (i.e., the wrinkling layer), where $\rho=1-(X / \Delta)$ with $X=\mathcal{O}(1)$. To this end we need to note that $\phi_{0}^{\prime}(1)=0$ (fixed), $\phi_{0}^{\prime \prime}(1)=1-\left(K_{0}^{2} / 2\right)$ (using the equation) and $\phi_{0}^{\prime \prime \prime}(1)=-\left(3+K_{0}^{2}\right)$ (differentiating the equation). Put together these results tell us that, where $X=\mathcal{O}(1)$, we have

$$
\Phi_{0}=\widetilde{\mu}^{2}\left[1+\frac{1}{4}\left(2-K_{0}^{2}\right)\left(\frac{X}{\Delta}\right)^{2}+\frac{1}{6}\left(3+K_{0}^{2}\right)\left(\frac{X}{\Delta}\right)^{3}+\ldots\right] .
$$


SM2.2. First order. At the next order, eliminating the $\Theta_{0}$ and $\Theta_{1}$ from the base equations (SM9b) and (SM10b) gives the equation for $\Phi_{1}$. By setting $\Phi_{1}=: \widetilde{\mu}^{2} \phi_{1}$ we obtain

$$
\phi_{1}^{\prime \prime}+\frac{1}{\rho} \phi_{1}^{\prime}-\frac{1}{\rho^{2}} \phi_{1}-\frac{K_{0}^{2} \rho^{3}}{\phi_{0}^{3}} \phi_{1}=-\frac{K_{0} \widetilde{\lambda}_{1} \rho^{3}}{\widetilde{\mu}^{3} \phi_{0}^{2}}, \quad \phi_{1}(0)=0, \quad \phi_{1}(1)=0 .
$$

Again, we need the Taylor series of $\phi_{1}$ where $X=\mathcal{O}(1)$. If we define the auxiliary problem

$$
\widetilde{\phi}_{1}^{\prime \prime}+\frac{1}{\rho} \widetilde{\phi}_{1}^{\prime}-\frac{1}{\rho^{2}} \widetilde{\phi}_{1}-\frac{K_{0}^{2} \rho^{3}}{\phi_{0}^{3}} \widetilde{\phi}_{1}=\frac{K_{0} \rho^{3}}{\phi_{0}^{2}}, \quad \widetilde{\phi}_{1}(0)=0, \widetilde{\phi}_{1}(1)=0
$$

then this is well-defined and with no parameters, as $K_{0}$ is already known. Standard numerical methods help us to identify $\widetilde{\phi}_{1}^{\prime}(1)=: K_{1}$, namely,

$$
K_{1} \simeq 0.5179 \text {. }
$$

Setting $\rho \rightarrow 1$ in the differential equation (SM15) also tells us that $\widetilde{\phi}_{1}^{\prime \prime}(1)=K_{0}-K_{1}$. Thus, the Taylor expansion of $\widetilde{\phi}_{1}$ as $\rho \rightarrow 1$ can be expressed in the form

$$
\widetilde{\phi}_{1} \rightarrow K_{1}\left(-\frac{X}{\Delta}\right)+\frac{1}{2}\left(K_{0}-K_{1}\right)\left(\frac{X}{\Delta}\right)^{2}+\ldots,
$$

so that

$$
\Phi_{1}=-\frac{\widetilde{\lambda}_{1}}{\widetilde{\mu}}\left[K_{1}\left(-\frac{X}{\Delta}\right)+\frac{1}{2}\left(K_{0}-K_{1}\right)\left(\frac{X}{\Delta}\right)^{2}+\ldots\right] .
$$

SM2.3. Second order. If we repeat the above procedures on (SM9c) and (SM10c) we find that, if $\Phi_{2}=: \widetilde{\mu}^{2} \phi_{2}$, then $\phi_{2}$ satisfies

$$
\phi_{2}^{\prime \prime}+\frac{1}{\rho} \phi_{2}^{\prime}-\frac{1}{\rho^{2}} \phi_{2}-\frac{K_{0}^{2} \rho^{3}}{\phi_{0}^{3}} \phi_{2}=-\frac{K_{0} \rho^{3} \widetilde{\lambda}_{2}}{\widetilde{\mu}^{3} \phi_{0}^{2}}-\frac{\widetilde{\lambda}_{1}^{2} \rho_{3}}{2 \widetilde{\mu}^{6} \phi_{0}^{2}}-\frac{3 K_{0}^{2} \rho^{3} \widetilde{\lambda}_{1}^{2} \widetilde{\phi}_{1}^{2}}{2 \widetilde{\mu}^{6} \phi_{0}^{4}}-\frac{2 \widetilde{\lambda}_{1}^{2} K_{0} \rho^{3} \widetilde{\phi}_{1}}{\widetilde{\mu}^{6} \phi_{0}^{3}},
$$

and restoring the original variables gives

(SM18)

$\Phi_{2}^{\prime \prime}+\frac{1}{\rho} \Phi_{2}^{\prime}-\frac{1}{\rho^{2}} \Phi_{2}-\frac{K_{0}^{2} \rho^{3}}{\phi_{0}^{3}} \Phi_{2}=-\frac{\widetilde{\lambda}_{2}}{\widetilde{\mu}}\left(\frac{\rho^{3} K_{0}}{\phi_{0}^{2}}\right)+\frac{\widetilde{\lambda}_{1}^{2}}{\widetilde{\mu}^{4}}\left[-\frac{\rho^{3}}{2 \phi_{0}^{4}}\left(\phi_{0}+K_{0} \widetilde{\phi}_{1}\right)\left(\phi_{0}+3 K_{0} \widetilde{\phi}_{1}\right)\right]$.

This must be solved subject to the homogeneous boundary conditions $\Phi_{2}(0)=\Phi_{2}(1)=$ 0 .

We can take advantage of the linearity of (SM18) and use the principle of superposition to solve it. The particular form of its right-hand side suggests introducing the auxiliary problems

$$
\Phi_{2 a}^{\prime \prime}+\frac{1}{\rho} \Phi_{2 a}^{\prime}-\frac{1}{\rho^{2}} \Phi_{2 a}-\frac{K_{0}^{2} \rho^{3}}{\phi_{0}^{3}} \Phi_{2 a}=\frac{\rho^{3} K_{0}}{\phi_{0}^{2}}, \quad \Phi_{2 a}(0)=\Phi_{2 a}(1)=0,
$$

and

(SM20)

$\Phi_{2 b}^{\prime \prime}+\frac{1}{\rho} \Phi_{2 b}^{\prime}-\frac{1}{\rho^{2}} \Phi_{2 b}-\frac{K_{0}^{2} \rho^{3}}{\phi_{0}^{3}} \Phi_{2 b}=\frac{\rho^{3}}{2 \phi_{0}^{4}}\left(\phi_{0}+K_{0} \widetilde{\phi}_{1}\right)\left(\phi_{0}+3 K_{0} \widetilde{\phi}_{1}\right), \quad \Phi_{2 b}(0)=\Phi_{2 b}(1)=0$. 
Note that $\Phi_{2}=\Phi_{2 a}+\Phi_{2 b}$ and we have already dealt with (SM19) in §SM2.2. Direct numerical integration of (SM20) immediately allows us to find $\Phi_{2 b}^{\prime}(1)=: K_{2}$, namely,

$$
K_{2} \simeq 0.0389 \text {. }
$$

Putting this together suggests that as $\rho \rightarrow 1$ so

$$
\Phi_{2}=\left(K_{1} \frac{\widetilde{\lambda}_{2}}{\widetilde{\mu}}+K_{2} \frac{\widetilde{\lambda}_{1}^{2}}{\widetilde{\mu}^{4}}\right)\left(\frac{X}{\Delta}\right)+\ldots .
$$

If we then combine the results (SM13), (SM17) and (SM22), we conclude that, where $X=\mathcal{O}(1)$, the base-state variable $\Phi$ assumes the behaviour

$$
\begin{aligned}
& \Phi=\Delta^{4} \widetilde{\mu}^{2}+\Delta^{2}\left[\frac{1}{4}\left(2-K_{0}^{2}\right) \widetilde{\mu}^{2} X^{2}+\frac{\widetilde{\lambda}_{1} K_{1}}{\widetilde{\mu}} X\right] \\
& +\Delta\left[\frac{1}{6}\left(3+K_{0}^{2}\right) \widetilde{\mu}^{2} X^{3}+\frac{\widetilde{\lambda}_{1}\left(K_{1}-K_{0}\right)}{2 \widetilde{\mu}} X^{2}+\left(K_{1} \frac{\widetilde{\lambda}_{2}}{\widetilde{\mu}}+K_{2} \frac{\widetilde{\lambda}_{1}^{2}}{\widetilde{\mu}^{4}}\right) X\right]+\mathcal{O}(1) .
\end{aligned}
$$

For simplicity, we shall define a sequence of constants $A_{i j}(i, j \in\{1,2,3\})$ so that this behaviour can be expressed more succinctly as

$(\mathrm{SM} 24) \quad \Phi=\widetilde{\mu}^{2} \Delta^{4}+\left[A_{22} X^{2}+A_{21} X\right] \Delta^{2}+\left[A_{13} X^{3}+A_{12} X^{2}+A_{11} X\right] \Delta+\mathcal{O}(1)$.

SM2.4. The bifurcation equation. We can use the information contained in (SM24) to deduce that for a wrinkle structure

$$
W=\widetilde{W}_{0}+\Delta^{-1} \widetilde{W}_{1}+\ldots \quad \text { and } \quad \Psi=\Delta^{-1} \widetilde{\Psi}_{0}+\Delta^{-2} \widetilde{\Psi}_{1}+\ldots,
$$

for some $W_{j}, \Psi_{j}$ (unknown at this stage). We note that at leading order in (SM3b) we just get

$$
\widetilde{M} \widetilde{\Psi}_{0}+2 K_{0} \widetilde{\mu}^{3} \widetilde{W}_{0}=0
$$

thus, the $\Psi_{j}$-functions are quite passive and respond to what the $W_{j}$-components need to do. At zeroth orders in the other equation $\left(O\left(\Delta^{6}\right)\right)$ we find that

$$
\mathcal{L}_{\#}\left[\widetilde{W}_{0}\right] \equiv \frac{d^{2} \widetilde{W}_{0}}{d X^{2}}-[\alpha X-\beta] \widetilde{W}_{0}=0,
$$

where

$$
\alpha:=-\frac{2 A_{22} \widetilde{M}}{\widetilde{\mu}^{2}} \quad \text { and } \quad \beta:=\frac{\left(A_{21}-\widetilde{M}\right) \widetilde{M}}{\widetilde{\mu}^{2}} .
$$

The solution of this equation is

$$
\widetilde{W}_{0} \propto \operatorname{Ai}\left(\alpha^{1 / 3}(X-\beta / \alpha)\right),
$$

which vanishes as $X \rightarrow 0$ if $-\beta / \alpha^{2 / 3}=-\zeta_{0}$, where $\zeta_{0} \simeq 2.331$. Making use of (SM27) this simplifies to

$$
\widetilde{\lambda}_{1} K_{1}=\widetilde{\mu} \widetilde{M}+2.331\left(\frac{1}{2} K_{0}^{2}-1\right)^{2 / 3} \widetilde{\mu}^{5 / 3} \widetilde{M}^{-1 / 3},
$$

which tells us that $\widetilde{\lambda}_{1}=\widetilde{\lambda}_{1}(\widetilde{M})$ has the property that $\widetilde{\lambda}_{1} \rightarrow+\infty$ as either $\widetilde{M} \rightarrow \infty$ or $\widetilde{M} \rightarrow 0^{+}$. Clearly, this indicates that the curve $\widetilde{\lambda}_{1}$ vs. $\widetilde{M}$ has a minimum, $\left(\widetilde{M}_{c}, \widetilde{\lambda}_{1 c}\right)$ (say), and simple numerical calculations yield

$$
\widetilde{M}_{c} \simeq 1.6877 \quad \text { and } \quad \widetilde{\lambda}_{1 c} \simeq 13.0346 .
$$


SM2.5. The higher-order correction term. At $\mathcal{O}\left(\Delta^{5}\right)$ in (SM3a) we obtain

$$
\begin{aligned}
& -X \widetilde{\mu}^{2} \frac{d^{2} \widetilde{W}_{0}}{d X^{2}}-\widetilde{\mu}^{2} \frac{d^{2} \widetilde{W}_{1}}{d X^{2}}-2 \widetilde{M} \frac{d^{2} \widetilde{W}_{0}}{d X^{2}}+2 \widetilde{M} X\left(\widetilde{M}-2 A_{22} X-A_{21}\right) \widetilde{W}_{0} \\
& +\widetilde{M}\left(2 \widetilde{M} X-3 A_{13} X^{2}-2 A_{12} X-A_{11}\right) \widetilde{W_{0}}+\widetilde{M}\left(\widetilde{M}-2 A_{22} X-A_{21}\right) \widetilde{W_{1}}=0 .
\end{aligned}
$$

If we recall the definitions of $\alpha$ and $\beta$ from (SM27) and work on the right-hand side of the above equation by using the governing equation for $\widetilde{W}_{0}$, we can re-cast (SM30) in the simplified form

$$
\mathcal{L}_{\#}\left[\widetilde{W}_{1}\right]=X \frac{d^{2} \widetilde{W}_{0}}{d X^{2}}-\frac{2 \widetilde{M}}{\widetilde{\mu}^{2}} \frac{d^{2} \widetilde{W}_{0}}{d X^{2}}+\frac{\widetilde{M}}{\widetilde{\mu}^{2}}\left(2 \widetilde{M} X-3 A_{13} X^{2}-2 A_{12} X-A_{11}\right) \widetilde{W}_{0}
$$

We only really need to work out how $\widetilde{W}_{1}$ behaves as $X \rightarrow 0$. However, before we can do that it is necessary to simplify further the right-hand side of (SM31). To this end, let us start by noting that equation (SM26) tells us that $X \widetilde{W}_{0}=\left(\widetilde{W}_{0}^{\prime \prime}+\beta \widetilde{W}_{0}\right) / \alpha$. Thus, reducing the $X^{2} \widetilde{W}_{0}$ by replacing one $X \widetilde{W}_{0}$ in this way gives

$$
\begin{aligned}
\mathcal{L}_{\#}\left[\widetilde{W}_{1}\right]=\left(1-\frac{3 \widetilde{M} A_{13}}{\widetilde{\mu}^{2} \alpha}\right) & X \frac{d^{2} \widetilde{W}_{0}}{d X^{2}}-\frac{2 \widetilde{M}}{\widetilde{\mu}^{2}} \frac{d^{2} \widetilde{W}_{0}}{d X^{2}} \\
+ & {\left[\frac{2 \widetilde{M}\left(\widetilde{M}-A_{12}\right)}{\widetilde{\mu}^{2}}-\frac{3 \beta \widetilde{M} A_{13}}{\widetilde{\mu}^{2} \alpha}\right] X \widetilde{W}_{0}-\frac{\widetilde{M} A_{11}}{\widetilde{\mu}^{2}} \widetilde{W}_{0} . }
\end{aligned}
$$

By differentiating $X \widetilde{W}_{0}=\left(\widetilde{W_{0}^{\prime \prime}}+\beta \widetilde{W}_{0}\right) / \alpha$ we have that $X \widetilde{W}_{0}^{\prime}=\left(\left(\widetilde{W}_{0}^{\prime \prime \prime}+\beta \widetilde{W}_{0}^{\prime}\right) / \alpha\right)-\widetilde{W}_{0}$ and $X \widetilde{W}_{0}^{\prime \prime}=\left(\left(\widetilde{W}_{0}^{\prime \prime \prime \prime}+\beta \widetilde{W}_{0}^{\prime \prime}\right) / \alpha\right)-2 \widetilde{W}_{0}^{\prime}$. Thus, the right-hand side of (SM32) becomes

$$
\begin{aligned}
R H S:=(1 & \left.-\frac{3 \widetilde{M} A_{13}}{\widetilde{\mu}^{2} \alpha}\right)\left[\frac{1}{\alpha}\left(\widetilde{W}_{0}^{\prime \prime \prime \prime}+\beta \widetilde{W_{0}^{\prime \prime}}\right)-2 W_{0}^{\prime}\right]-\frac{2 \widetilde{M}}{\widetilde{\mu}^{2}} \frac{d^{2} \widetilde{W}_{0}}{d X^{2}} \\
+ & {\left[\frac{2 \widetilde{M}\left(\widetilde{M}-A_{12}\right)}{\widetilde{\mu}^{2}}-\frac{3 \beta \widetilde{M} A_{13}}{\widetilde{\mu}^{2} \alpha}\right] \frac{1}{\alpha}\left(\widetilde{W}_{0}^{\prime \prime}+\beta \widetilde{W}_{0}\right)-\frac{\widetilde{M} A_{11}}{\widetilde{\mu}^{2}} \widetilde{W}_{0} . }
\end{aligned}
$$

Now the RHS is expressed as a linear multiple of various derivatives of $\widetilde{W}_{0}$. To write down the solution of $\mathcal{L}_{\#}\left[\widetilde{W}_{1}\right]=R H S$, we need the following observation. If we denote by $g^{(n)}$ the $n^{t h}$-order derivative of the function $g \equiv g(X)(n \in \mathbb{N})$, then the particular integral of the equation in $f \equiv f(X)$,

$$
\mathcal{L}_{\#}[f]=g^{(n)},
$$

is given by

$$
f=f_{\mathrm{part}}(X):=\frac{1}{(n+1) \alpha} g^{(n+1)}(X) .
$$


Given these obsevations we can now write down the solution of the full $\widetilde{W}_{1}$ equation. Putting everything together, we finally get

$$
\begin{aligned}
\widetilde{W}_{1}= & \left(1-\frac{3 \widetilde{M} A_{13}}{\widetilde{\mu}^{2} \alpha}\right)\left[\frac{1}{\alpha^{2}}\left(\frac{1}{5} \widetilde{W}_{0}^{(5)}+\frac{1}{3} \beta \widetilde{W}_{0}^{\prime \prime \prime}\right)-\frac{1}{\alpha} \widetilde{W_{0}^{\prime \prime}}\right]-\frac{2 \widetilde{M}}{\widetilde{\mu}^{2}} \frac{\widetilde{W}_{0}^{\prime \prime \prime}}{3 \alpha} \\
& +\left[\frac{2 \widetilde{M}\left(\widetilde{M}-A_{12}\right)}{\widetilde{\mu}^{2}}-\frac{3 \beta \widetilde{M} A_{13}}{\widetilde{\mu}^{2} \alpha}\right] \frac{1}{\alpha^{2}}\left(\frac{1}{3} \widetilde{W}_{0}^{\prime \prime \prime}+\beta \widetilde{W_{0}^{\prime}}\right)-\frac{\widetilde{M} A_{11} \widetilde{W}_{0}^{\prime}}{\widetilde{\mu}^{2} \alpha}
\end{aligned}
$$

Let us recall that we are solely interested in what happens to (SM34) as $X \rightarrow 0$. We already know that $\widetilde{W}_{0}(0)=0$, and we set $\widetilde{W}_{0}^{\prime}(0)=: \omega_{0}$. In light of this notation the governing equation (SM26) and its differential consequences imply that

$$
\widetilde{W}_{0}^{\prime \prime}(0)=0, \quad \widetilde{W}_{0}^{\prime \prime \prime}(0)=-\beta \omega_{0}, \quad \widetilde{W}_{0}^{(4)}(0)=2 \alpha \omega_{0}, \quad \widetilde{W}_{0}^{(5)}(0)=\beta^{2} \omega_{0} .
$$

Together with (SM34) this then leads us to

$$
\widetilde{W}_{1} \rightarrow\left[-\frac{2 \beta^{2}}{15 \alpha^{2}}-\frac{8 \beta^{2} A_{13} \widetilde{M}}{5 \widetilde{\mu}^{2} \alpha^{3}}+\frac{2 \widetilde{M} \beta}{3 \alpha \widetilde{\mu}^{2}}+\frac{4 \widetilde{M} \beta\left(\widetilde{M}-A_{12}\right)}{3 \alpha^{2} \widetilde{\mu}^{2}}-\frac{\widetilde{M} A_{11}}{\alpha \widetilde{\mu}^{2}}\right] \omega_{0}, \quad \text { as } \quad X \rightarrow 0 .
$$

SM3. The bending layer. To tie things down we still need to consider the rim bending layer where all the boundary conditions on the perturbation are imposed. It can be shown by easy balances that the depth of the inner zone is $\mathcal{O}\left(\Delta^{-2}\right)$, so we are led to introduce a new rescaled variable $\zeta=\mathcal{O}(1)$ defined by

$$
\rho=1-\frac{\zeta}{\Delta^{2}}
$$

We are somewhat fortunate as this happens to be the rim layer for the base state as well. This layer only operates on the $\Theta$ component and that is just too small to come into play (so the driving differential operator in the bending layer will have constant coefficients). It turns out that the leading-order equation for the $W$-component of the wrinkle is just

$$
\frac{d^{4} W_{\text {bend }}}{d \zeta^{4}}-\mu^{2} \frac{d^{2} W_{\text {bend }}}{d \zeta^{2}}=0 .
$$

We need the solution of this differential equation to match onto the linearly decaying $\widetilde{W}_{0}$ as $\zeta \rightarrow \infty$, and to satisfy the rim conditions that $W_{\text {bend }}$ and its first derivative vanish on $\zeta=0$; these constraints leave us with

$$
W_{\text {bend }}=\zeta+\frac{1}{\widetilde{\mu}} \exp (-\widetilde{\mu} \zeta)-\frac{1}{\widetilde{\mu}} .
$$

So this tells us that $W_{\text {bend }}$ grows like $\zeta$, while the constant part of its large- $\zeta$ behaviour is simply $-1 / \widetilde{\mu}$. We can now take advantage of these observations in conjunction with (SM35) to deduce that

$$
-\frac{2 \beta^{2}}{15 \alpha^{2}}-\frac{8 \beta^{2} A_{13} \widetilde{M}}{5 \widetilde{\mu}^{2} \alpha^{3}}+\frac{2 \widetilde{M} \beta}{3 \alpha \widetilde{\mu}^{2}}+\frac{4 \widetilde{M} \beta\left(\widetilde{M}-A_{12}\right)}{3 \alpha^{2} \widetilde{\mu}^{2}}-\frac{\widetilde{M} A_{11}}{\alpha \widetilde{\mu}^{2}}=-\frac{1}{\widetilde{\mu}},
$$

whence, by re-arrangement,

$$
\widetilde{\lambda}_{2}=\frac{\widetilde{\mu}}{K_{1}}\left[\frac{1}{\widetilde{M}}\left(\widetilde{\mu} \alpha-\frac{2 \beta^{2} \widetilde{\mu}^{2}}{15 \alpha}\right)-\frac{8 A_{13} \beta^{2}}{5 \alpha^{2}}+\frac{2 \beta}{3}+\frac{4 \beta\left(\widetilde{M}-A_{12}\right)}{3 \alpha}-\frac{\widetilde{\lambda}_{1}^{2} K_{2}}{\widetilde{\mu}^{4}}\right]
$$


Since by our original assumption (SM6) $\widetilde{\mu}=\mathcal{O}(1)$, we are free to set $\widetilde{\mu}=1$ in (SM37). Substituting also the numerical values (SM12), (SM16), (SM21) and (SM29) we eventually get

(SM38)

$$
\widetilde{\lambda}_{2} \simeq 54.8417
$$

\section{REFERENCES}

[SM1] C.D. Coman, AmD A.P. Bassom, Wrinkling structures at the rim of an initially stretched circular thin plate subjected to transverse pressure, Submitted. 